\title{
Das Kompetenz-Reflexions-Inventar (KRI) - Konstruktion und erste psychometrische Überprüfung eines Messinstrumentes
}

\author{
Simone Kauffeld ${ }^{1}$ \\ Angenommen: 28. April 2021 / Online publiziert: 30. Juni 2021
}

(c) Der/die Autor(en) 2021

\section{Zusammenfassung}

Dieser Beitrag der Zeitschrift „Gruppe. Interaktion. Organisation.“ (GIO) beschäftigt sich mit dem Kompetenz-Reflexions-Inventar (KRI), welches das Selbstkonzept beruflicher Handlungskompetenz erfasst. Der Fragebogen besteht aus 80 Items, die in $20 \mathrm{~min}$ beantwortet werden können. Mit Hilfe von separaten Faktorenanalysen $(N=156)$ konnten für die Fachkompetenz die Skalen Konzeptionelles Denken, Kenntnis der Organisation und Abläufe, Problemerkennung, Entwicklung von Lösungen, für die Methodenkompetenz die Skalen Moderation und Präsentation, Planung, Reflexivität, Umgang mit IKT (Informations- und Kommunikationstechnologie) und Technik, Konzentration, Aufgabenverteilung, für die Sozialkompetenz die Skalen Positionierung des eigenen Standpunktes, Motivation von Anderen, Akzeptanz und Anerkennung, Kontaktaufbau und -pflege, Rücksichtnahme und Besonnenheit und für die Selbstkompetenz die Skalen Mitwirkung, Verantwortungsübernahme und Initiative, Veränderungsinteresse sowie Selbstmanagement identifiziert und an einer zweiten Stichprobe weitgehend bestätigt werden $(N=368)$. Konfirmatorische Faktorenanalysen ergaben darüber hinaus Hinweise für eine Einteilung der 19 Skalen beruflicher Handlungskompetenz in die vier Facetten Fach-, Methoden-, Sozialund Selbstkompetenz. Als Validitätshinweise können die moderaten bis mittleren positiven Zusammenhänge der Kompetenzskalen und -facetten zum (1) beruflichen Erfolg, (2) dem Brutto-Jahres-Einkommen, (3) zur Zufriedenheit im Beruf sowie (4) den lernrelevanten Merkmalen der Arbeitsaufgabe interpretiert werden. Die Veränderungssensitivität des KRI bei Trainingsmaßnahmen konnte in zwei Teilstichproben nachgewiesen werden. Zusammenfassend ermöglicht das KRI, das hinsichtlich zentraler Gütekriterien an Berufstätigen überprüft wurde, die standardisierte und ökonomische Erfassung des Selbstkonzepts beruflicher Kompetenz.

Schlüsselwörter Kompetenz $\cdot$ Kompetenzdiagnose $\cdot$ Personalentwicklung $\cdot$ Selbstkonzept

\section{The competence-reflection-inventory (CRI)—Development and first psychometric validation of an instrument for measuring the self-concept of competence.}

\footnotetext{
Abstract

This article in the journal "Gruppe. Interaktion. Organisation." (GIO) deals with the Competence-Reflection-Inventory (KRI), that measures the self-concept of competence. The questionnaire consists of 80 items which can be answered within $20 \mathrm{~min}$.
}

Univ.-Prof. Dr. Simone Kauffeld

s.kauffeld@tu-braunschweig.de

1 Arbeits-, Organisations- und Sozialpsychologie, Institut für Psychologie, TU Braunschweig, Spielmannstr. 19, 38106 Braunschweig, Deutschland 
Separate factor analyses $(N=156)$ identified the following scales for professional competence: conceptual thinking, organizational/process knowledge, problem perception, and solution development; for methodological competence: presentation, scheduling, reflectivity, EDP/technology handling, concentration, and task assignment, for social competence: positioning one's point of view, motivating others, acceptance/acknowledgement, initiation/maintenance of contacts, and considerateness, and for personal competence: participation, assuming responsibility/initiative, interest in change, and self management. These scales were largely replicated in a second sample $(N=368)$. In addition, confirmatory factor analyses supported categorization of the 19 scales into the four facets professional, methodological, social, and personal competence. The following moderate to medium positive correlations between the competence scales and (1) professional success, (2) gross annual income, (3) job satisfaction, and (4) task characteristics relevant for learning, can be interpreted as evidence for validity. Trainings with two partial samples indicated that the KRI is susceptible to change. In sum, the KRI was validated concerning central psychometric properties. It thus enables standardized and economic assessment of the self concept of work-related competence.

Keywords Competence · Competence diagnosis · Human resources development

Moderne Arbeitswelten erfordern neue Kompetenzen von Beschäftigten (Helmrich et al. 2016). Lebenslanges Lernen ist bedeutsame Aufgabe im 21. Jahrhundert, um langfristig die Beschäftigungsfähigkeit zu sichern (Van Laar et al. 2017). Mitarbeitende lernen nicht nur innerhalb formalisierter Weiterbildung und Personalentwicklungsmaßnahmen, sondern auch im Prozess der Arbeit (Decius et al. 2019; Kortsch et al. 2019; Kauffeld 2016) und erhalten so ihre Beschäftigungsfähigkeit (Gerken et al. 2016). Sie entwickeln dabei berufliche Handlungskompetenzen, die ihnen helfen, vertraute wie auch neue Aufgaben zu bewältigen. Der Erhalt und die Weiterentwicklung der beruflichen Handlungskompetenzen sind zentrale Aspekte des lebenslangen Lernens.

Die Erfassung und Beurteilung von Kompetenzen kann als Voraussetzung für die Entwicklung beruflicher Handlungskompetenz des Einzelnen angesehen werden. In vielen Unternehmen werden unternehmensspezifische Kompetenzmodelle und -managementsysteme etabliert (Kauffeld und Paulsen 2018). Neben unternehmensspezifischen Kompetenzanforderungen können jedoch auch generische Kompetenzanforderungen beschrieben werden, die über verschiedene Organisation hinweg gelten (Soderquist et al. 2010). Ein Beispiel für ein generisches Kompetenzmodell stellen die Great Eight dar, die acht Kompetenzcluster beschreiben (Bartram 2005). Im deutschsprachigen Raum findet sich häufig eine Unterteilung in Fach-, Methoden-, Sozial- und Selbstkompetenzfacetten. Bestehende Instrumente decken hier jedoch nicht alle Facetten ab (z.B. SBK, Sonntag und Schäfer-Rauser 1993; ISK, Kanning 2009, 2014).

Ziel des vorliegenden Artikels ist es, die Entstehung und erste psychometrische Überprüfung eines Instruments zur differenzierten und ökonomischen Erfassung des Selbstkonzepts beruflicher Handlungskompetenz aller vier Facetten darzustellen. Als Einsatzfeld wird die Personalentwicklung und Karriereberatung fokussiert.

\section{Definition von Kompetenz}

Die berufliche Handlungskompetenz umfasst alle Fähigkeiten, Fertigkeiten, Denkmethoden und Wissensbestände des Menschen, die ihn bei der Bewältigung konkreter sowohl vertrauter als auch neuartiger Arbeitsaufgaben selbstorganisiert, aufgabengemäß, zielgerichtet, situationsbedingt und verantwortungsbewusst - oft in Kooperation mit anderen handlungs- und reaktionsfähig machen und sich in der erfolgreichen Bewältigung konkreter Arbeitsanforderungen zeigen (Kauffeld 2006a; vgl. auch Weinert 2001). Im Folgenden wird einer Klassifikation der beruflichen Handlungskompetenz in die vier Kompetenzbereiche Fach-, Methoden-, Sozial- und Selbstkompetenz gefolgt. Diese Unterteilung aus der Berufsbildungspraxis weist deutliche Analogien $\mathrm{zu}$ theoretisch fundierten Systematisierungen von Lernergebnissen bei beruflichen Trainingsmaßnahmen in der anglo-amerikanischen Literatur auf (vgl. Kraiger et al. 1993) und hat sich in der Praxis weitgehend durchgesetzt (vgl. Sonntag und Schaper 1999; Sonntag 2004; Sonntag und Schmidt-Rathjens 2004; Kauffeld und Albrecht 2021).

Unter Fachkompetenzen sind organisations-, prozess-, aufgaben- und arbeitsplatzspezifische berufliche Fertigkeiten und Kenntnisse zu verstehen sowie die Fähigkeit, organisationales Wissen sinnorientiert einzuordnen und zu bewerten, Probleme zu identifizieren und Lösungen zu generieren. Methodenkompetenzen beschreiben situationsübergreifend und flexibel eingesetzte kognitive Fähigkeiten zum Beispiel zur Problemstrukturierung oder Entscheidungsfindung. Alle Dispositionen, Handlungen kommunikativ und kooperativ selbstorganisiert vorzunehmen, sowie Interaktionssituationen mit Kollegen, Vorgesetzten, Mitarbeitern, Kunden oder Geschäftspartnern zur Entwicklung und Umsetzung von Plänen und Zielen nutzen zu können, werden der Sozialkompetenz zugeordnet. Unter die Selbstkompetenz fallen Fähigkeiten zur Selbstwahrnehmung, Bewer- 
tung eigener Handlungen und bewussten Reflexion der eigenen Fähigkeiten. Selbstkompetente Mitarbeiter sind offen für Veränderungen, haben Interesse aktiv zu gestalten und schaffen sich eigeninitiativ Möglichkeiten zur Mitwirkung (vgl. Kauffeld 2006a).

Einigkeit herrscht weitgehend, dass die Kompetenzfacetten Fach-, Methoden-, Sozial- und Selbstkompetenz nicht unabhängig voneinander sind. Um z. B. aktiv gestalten zu können, wird es hilfreich sein, andere einzubinden und für die eigenen Ideen zu begeistern (Sozialkompetenz). Der Einsatz von Methoden zur Strukturierung kann z.B. helfen, fachliche Kompetenzen zum Tragen kommen zu lassen. Aus der Verknüpfung der Kompetenzen ergibt sich die spezifisch ausgeprägte berufliche Handlungskompetenz (vgl. ausführlich Kauffeld 2016; Kauffeld und Paulsen 2018).

\subsection{Anforderungen an ein Kompetenzmessinstrument}

Ein Instrument zur Kompetenzmessung muss Anforderungen, die sich aus dem Kompetenzverständnis ergeben genauso genügen, wie psychometrischen Gütekriterien und Erwägungen aus der Praxis (vgl. Kauffeld 2006a; Kauffeld und Paulsen 2018).

Kompetenzverständnis Die Entwicklung eines Kompetenzmessverfahrens muss am Kompetenzbegriff ansetzen. Kompetenzen sind an die erfolgreiche Bewältigung von hinlänglich komplexen Anforderungen gebunden, d.h. sie müssen sich an Standards des Erfolgs bzw. der Zielerreichung messen lassen. Die Kompetenzen sollten unabhängig vom Erwerbskontext erfasst werden. Um Aussagen über das Zusammenspiel der vier Facetten machen zu können, sollten sie mit einem Verfahren abbildbar sein. Aus der Kompetenzdiagnose sollten sich Kompetenzentwicklungsmaßnahmen ableiten lassen. Darüber hinaus sollte ein Kompetenzdiagnoseinstrument veränderungssensitiv sein, d.h. Kompetenzzuwächse bzw. -verluste abbilden können, um Entwicklungsschritte dokumentieren zu können.

Gütekriterien Ein Kompetenzmessverfahren sollte den „,klassischen“ Gütekriterien der psychologischen Testtheorie (Objektivität, Reliabilität, Validität) genügen.

Praxis Mit dem Einsatz des Messinstruments muss ein Nutzen für bestimmte Personen oder Organisationen verbunden sein. Eine Kompetenzmessung sollte fair sein und allen Kandidaten die gleichen Chancen geben, sich durch Transparenz auszeichnen und eine Ergebnisrückmeldung enthalten. Die Kompetenzmessung muss organisierbar sein und finanzierbar bleiben (Sach-, Zeit- und Personalaufwand). Für vergleichende Analysen sollte es möglichst breit anwend- bar sein, z.B. über Berufsgruppen und Hierarchieebenen hinweg.

\subsection{Zugänge zur Kompetenzmessung}

Fünf Zugänge zur Kompetenzmessung können unterschieden werden (vgl. ausführlich Kauffeld 2006a). Während (1) ökonomisch-orientierte Verfahren eine betriebswirtschaftliche Bewertung des Personals anstreben, strukturiert (2) der tätigkeitsanalytische Ansatz berufliche Handlungssituationen, um die Ableitung arbeitsorganisatorischer und qualifikatorischer Konsequenzen zu ermöglichen. Ein Beispiel hierfür ist die Erstellung von Teiltätigkeitslisten (z. B. Frieling et al. 2003). (3) Biografische Ansätze, die Kompetenzen aus bisherigen Leistungen und erworbenen Qualifikationen ableiten (z.B. Kompetenzbiografie; Erpenbeck und Heyse 1999), sind von (4) persönlichkeitsorientierten Ansätzen zu unterscheiden bei denen situationsabhängige und stabile Kompetenzen erfasst werden. (5) Innerhalb des verhaltensanalytischen Modells wird die Situationsabhängigkeit kompetenten Verhaltens betont. Personen verfügen demnach über berufliche Handlungskompetenz, wenn sie konkrete berufliche Situationen erfolgreich bewältigen können. Diese Vorstellung entspricht dem dargestellten Kompetenzverständnis am besten. Wie können Kompetenzen im verhaltensanalytischen Modell gemessen werden? Neben der Beobachtung im natürlichen oder künstlichen Setting, können Befragungen vorgenommen werden. Beobachtung und Befragung können als Fremd- oder Selbsteinschätzung erfolgen (zu Vor- und Nachteilen der verschiedenen Ansätze vgl. ausführlich Kauffeld 2006a; Kauffeld und Paulsen 2018).

Die Selbstbeschreibungsverfahren erlauben die standardisierte und vergleichsweise ökonomische Erfassung von Kompetenzen. Die Erhebung des Selbstkonzepts beruflicher Kompetenz, die mit dem Kompetenz-Reflexions-Inventar verfolgt wird, geht davon aus, dass die Befragten Experten ihrer Tätigkeit sowie ihrer selbst sind und damit eine Einschätzung des eigenen arbeitsbezogenen Wissens und Könnens möglich wird (Bergmann 2000; Hacker 1998). Selbstbeschreibungen können beim Einzelnen Reflexionsprozesse anstoßen, die eigene Erfolgszuschreibung fördern, den eigenen Kompetenzentwicklungsbedarf bewusst machen, die realistische Zielsetzung unterstützen und Wege für die Zielerreichung aufzeigen (Herold 2003). Individuelle Fortschritte können beschrieben werden. Eine Einschätzung der eigenen Kompetenzen ermöglicht eine Orientierung bei selbstselektiven Entscheidungen. Die Selbstevaluation beruflicher Kompetenz kann Personalbeurteilungsgesprächen vorgeschaltet werden und die Akzeptanz der daraus abgeleiteten Urteile steigern (vgl. zusammenfassend Kauffeld 2006a). Eine Einschätzung der eigenen Kompetenzen er- 
möglicht eine Orientierung bei selbstselektiven Entscheidungen.

\section{Fragebogen zum Selbstkonzept beruflicher Kompetenz}

Es existieren bereits mehrere Selbstbeschreibungsverfahren (vgl. zusammenfassend Erpenbeck und v. Rosenstiel 2003; Kanning und Holling 2002; Sarges und Wottawa 2001; Erpenbeck et al. 2017). Sonntag und Schmidt-Rathjens (2004) resümieren kritisch, dass viele Instrumente nicht frei zugänglich sind und Vermarktungsinteressen dominieren. Die meisten Verfahren stellen lediglich erste Ansätze dar, deren weitere Erprobung noch aussteht. Die in der Diagnostik üblichen Konstruktions- und Validierungsanforderungen werden dabei selten erfüllt.

Bei den Verfahren, die als psychometrischen überprüft gelten können, wie der Fragebogen zum Selbstkonzept beruflicher Kompetenzen (SBK; Sonntag und Schäfer-Rauser 1993), der Beurteilungsbogen zu sozialen und methodischen Kompetenzen (smk; Frey und Balzer 2005) sowie das Inventar Sozialer Kompetenzen (ISK; Kanning 2009, 2014) werden nur drei bzw. zwei oder auch nur eine der überfachlichen Kompetenzfacetten erfasst. Die ersten beiden Verfahren sind zudem auf die Kompetenzmessung bei Auszubildenden zugeschnitten und nur hier psychometrisch überprüft, was ihre Anwendungsbreite stark einschränkt. Darüber hinaus sind zahlreiche weitere Selbst- und Fremdbeschreibungsbogen für bestimmte Zielgruppen entstanden (z.B. für Trainer und Trainierinnen [Grohmann et al. 2021]).

Zusammenfassend ist festzuhalten, dass kein Verfahren zur differenzierten Erfassung der vier Kompetenzfacetten Fach-, Methoden-, Sozial- und Selbstkompetenz zur Verfügung steht. Ferner gibt es keine einheitliche Differenzierung von Kompetenzaspekten innerhalb der Facetten. Je spezifischer Kompetenz-Stärken und -Schwächen jedoch diagnostiziert werden können, desto gezielter können geeignete Maßnahmen angesetzt werden.

\subsection{Fragestellungen}

Da es keine allgemeingültige Unterteilung der vier Kompetenzfacetten in verschiedene Skalen gibt, soll zunächst der Frage nachgegangen werden, welche Dimensionen sich innerhalb der Fach-, Methoden-, Sozial- und Selbstkompetenz identifizieren lassen. Die ermittelten Skalen des Fragebogens gilt es in der zweiten Stichprobe zu replizieren.

Darüber hinaus wird davon ausgegangen, dass die berufliche Handlungskompetenz in die vier Kompetenzfacetten Fach-, Methoden-, Sozial- und Selbstkompetenz unterteilt werden kann. Da andere Autoren Fach- und Methodenkom- petenz nicht unterteilen (z. B. Erpenbeck und v. Rosenstiel 2003) oder Methoden-, Sozial- und Selbstkompetenz zur überfachlichen Kompetenz zusammenfassen (z.B. Reisse 1995), sollen zwei und drei Faktoren-Modelle alternativ getestet werden. Die Modelle mit korrelierten Faktoren sollen gemäß der Annahme, dass die Facetten nicht unabhängig voneinander sind, einen besseren Fit aufweisen als die Modelle mit orthogonalen Faktoren.

Der Kompetenzdefinition folgend, sollten Kompetenzen sich in der erfolgreichen Bewältigung von Arbeitsaufgaben zeigen. Daher wird angenommen, dass die Kompetenzen mit dem beruflichen Erfolg in Zusammenhang stehen (vgl. auch Miles 1965; zur Empirie vgl. Frey und Balzer 2005; Judge et al. 1995; HayGroup 2004). Dem Human Resources Model (HRM; Miles 1965) folgend, sollten kompetente Mitarbeiter zufriedener sein, weil Kompetenzen zu höheren Leistungen führen und die Mitarbeiter zufriedener sind, wenn sie den Erfolg auf ihre Leistung zurückführen. Metaanalytisch ließ sich zeigen, dass zwischen dem Selbstkonzept eines Individuums und der Arbeitszufriedenheit moderate positive Korrelationen bestehen (Judge und Bono 2001).

Kompetenzentwicklung kann formell in Seminaren und Schulungen oder informell durch das Lernen in der Arbeit gefördert werden (vgl. Kauffeld und Paulsen 2018). Grundlage für die arbeitsimmanente Kompetenzentwicklung der Mitarbeiter bilden Lernpotenziale der Arbeit. In mehreren Untersuchungen konnte gezeigt werden, dass die Gestaltung lernrelevanter Arbeitsmerkmale Einfluss auf die Kompetenzentwicklung nimmt (z.B. Schaper 2000; Wardanjan 2000; Wieland 2004; Kauffeld 2006b; Decius et al. 2019; Rowold und Kauffeld 2009). Gleichzeitig kann auch davon ausgegangen werden, dass kompetente Mitarbeitende, die ein hohes Maß an Gestaltungskompetenz als Aspekt der Selbstkompetenz mitbringen durch job crafting (vgl. Rudolph et al. 2017) ihre Arbeit lernförderlich gestalten. Daher kann unabhängig von der Wirkrichtung angenommen werden, dass ein positiver Zusammenhang zwischen der Lernförderlichkeit der Arbeitsaufgabe und den Kompetenzfacetten der beruflichen Handlungskompetenz besteht.

Da Kompetenzen durch Trainingsmaßnahmen verbessert werden können (vgl. Metaanalyse von Arthur et al. 2003), sollten diese Veränderungen mit dem KRI abbildbar sein. Unterschiede sollten sich vorrangig hinsichtlich der Kompetenzfacetten zeigen, auf deren Entwicklung die Intervention ausgerichtet ist (vgl. Sonntag und Schaper 1999). Ein Stationsleitungskurs sollte Auswirkungen auf die vier Kompetenzfacetten zeigen. Ein Self-Assessment-Training, das auf die Einschätzung der eigenen Stärken und Schwächen abzielt, sollte sich primär in der Selbstkompetenz niederschlagen. 


\subsection{Methode}

\subsubsection{Stichproben}

Erste Stichprobe Die erste Stichprobe setzt sich aus 156 Berufstätigen aus verschiedenen Organisationen unterschiedlicher Branchen (v.a. Automobilindustrie, Verpackungsindustrie, öffentlicher Dienst und Verwaltung), die über persönliche Kontakte rekrutiert wurden, zusammen. Das Durchschnittsalter der Berufstätigen beträgt $M=38,6$ Jahre $(S=10)$. Mit $n=78$ Frauen kann das Verhältnis von Männern zu Frauen in der Stichprobe als ausgewogen bezeichnet werden. Bei den Personen die Angaben machten $(N=150)$, verfügten $38,5 \%$ über eine abgeschlossene Lehre und 51,3\% über einen (Fach-) Hochschulabschluss als höchstem Bildungsabschluss. Die Anzahl der Berufsjahre reicht von 0,3 bis 38 Jahre $(M=14,7$ Jahre, $S D=10,3$ Jahre), die Tätigkeitsdauer variierte zwischen 0,3 bis 36 Jahren ( $M=7,1$ Jahre, $S D=7,4$ Jahre).

Die Teilnahme an der Untersuchung war für alle Untersuchungsteilnehmenden grundsätzlich freiwillig. Den Teilnehmern wurde in dieser wie auch in der zweiten Stichprobe als Gegenleistung für ihre Teilnahme ein KompetenzCheck, d.h. eine schriftliche Rückmeldung ihrer Kompetenzen angeboten. Bei den Wiederholungsmessungen wurde die Rückmeldung nach der zweiten Messung angeboten.

Zweite Stichprobe Die zweite Stichprobe $(N=368)$, in der die Endfassung des KRI eingesetzt wurde, setzt sich zu fast $90 \%$ aus dem Dienstleitungsbereich (Gesundheit, Finanzdienstleistung, Sicherheit) zusammen. Das Durchschnittsalter der Personen die persönliche Angaben machten $(N=227)$, beträgt $M=35,8$ Jahre $(S=9,7)$. Mit 52,8\% Frauen kann das Verhältnis von Männern zu Frauen auch in der zweiten Stichprobe als ausgewogen bezeichnet werden. Bei den Personen die Angaben machten, verfügten 42,3\% über eine abgeschlossene Lehre und 55,5\% über einen (Fach-) Hochschulabschluss als höchstem Bildungsabschluss.

Um feststellen zu können, ob das KRI in der Lage ist, Veränderungen der Kompetenzen abzubilden, wurde in den beiden Teilstichprobe "Stationsleitung in der Pflege“ und „Auszubildende eines Finanzdienstleistungsunternehmens“ eine Messwiederholung durchgeführt.

Die Teilstichprobe Stationsleitung umfasst 63 Pflegekräfte aus mehreren Krankenhäusern, die in drei Gruppen an einem Stationsleitungskurs teilnahmen. 62 Personen (98,4\%) machten Angaben zu ihrem Alter. Das Durchschnittsalter betrug $M=36,8$ Jahre $(S=6,6)$. Mit $n=42$ $(66,7 \%)$ Frauen und $n=21(33,3 \%)$ Männern überwiegt in dieser Stichprobe der Anteil der Frauen. 51 Personen $(80,9 \%)$ verfügten über eine abgeschlossene Lehre, elf Teilnehmer $(17,5 \%)$ über eine universitäre Ausbildung. Von zwei Trainingsgruppen $(n=40)$ liegen Messwiederholungs- daten vor. In der Teilstichprobe Stationsleitung besuchten die Pflegekräfte zwischen den beiden Messzeitpunkten einen Stationsleitungskurs. Der Stationsleiterkurs gliedert sich in sechs Blöcke von jeweils zweiwöchiger Dauer in einem Zeitraum von acht Monaten und umfasst insgesamt 500 Unterrichtsstunden. Inhalte des Kurses sind: Führen von Mitarbeitern, Management von Pflegequalität, Rechtskunde, Betriebswirtschaftslehre sowie die Organisation der Stationsarbeit. Im Verlauf des Kurses werden Lernziele gesetzt und Lernaufträge formuliert. Die erste Messung erfolgte zu Beginn, die zweite am Ende des Kurses.

Die Teilstichprobe „Auszubildende Finanzdienstleitung“ setzt sich aus 37 Auszubildenden im dritten Ausbildungsjahr eines Finanzdienstleistungsunternehmens zusammen. 18 Auszubildende nahmen an einem einwöchigen Self-Assessment teil ${ }^{1}$. Eine Kontrollgruppe von 19 Auszubildenden erhielt kein Training. Das Durchschnittsalter der Auszubildenden betrug $M=21,5$ Jahre $(S=3,0)$. Unter den Auszubildenden waren $n=20$ Personen $(54,1 \%)$ weiblich. In der Teilstichprobe des Finanzdienstleistungsunternehmens konnte ein quasiexperimentelles Untersuchungsdesign mit Kontrollgruppe realisiert werden. In dem Self-Assessment standen die Bilanzierung eigener Stärken und Schwächen vor allem anhand von Assessment-Center-Übungen mit am Kompetenzmodell des Unternehmens orientierten Feedback aus der Gruppe und Überlegungen zur weiteren beruflichen Entwicklung im Mittelpunkt. Die erste Messung mit dem KRI wurde unmittelbar vor dem Self-Assessment, die zweite Messung zwei Monate danach vorgenommen.

\section{Messinstrumente}

Brutto-Jahres-Gehalt Das Brutto-Jahres-Gehalt (incl. Zusatzleistungen wie Dienstwagen etc.) wurde als objektives Validierungskriterium $(M=43.965,39 € ; S D=24.902,92 €)$ von den Mitarbeitern erfragt. Von 110 Personen der ersten Stichprobe (70,5\%) liegen hierzu Angaben vor.

Beruflicher Erfolg Der Berufliche Erfolg wurde mit zwei Items (Beispiel: Ich bin in meinem Beruf sehr erfolgreich.) erhoben $(M=72,11 ; S D=18,34 ; \alpha=0,83)$.

Zufriedenheit im Beruf Die Zufriedenheit im Beruf wurde mit zwei Items (Beispiel: Meine berufliche Tätigkeit gefällt mir sehr) gemessen $(M=75,68 ; S D=21,09 ; \alpha=0,94)$.

Lernrelevante Merkmale der Arbeitsaufgabe Der Fragebogen $\mathrm{zu}$ lernrelevanten Merkmalen der Arbeitsaufgabe

\footnotetext{
1 Von einem Teilnehmer der Interventionsgruppe liegen nur unvollständige Daten vor, deshalb wurde dieser Datensatz aus den nachfolgenden Analysen ausgeschlossen.
} 
Tab. 1 Validierungsskalen und ihre Interkorrelationen

\begin{tabular}{llllll}
\hline Skala & Item-anzahl & 1 & 2 & 3 & 4 \\
\hline 1. Brutto-Jahres-Gehalt & 1 & - & - & - & - \\
2. Beruflicher Erfolg & 2 & $0,35^{* *}$ & $(0,83)$ & - & - \\
3. Zufriedenheit im Beruf & 2 & $0,25^{* *}$ & $0,72^{* *}$ & $(0,94)$ & - \\
4. Lernrelevante Merkmale der Arbeitsaufgabe & 24 & $0,33^{* *}$ & $0,67^{* *}$ & $0,62^{* *}$ & $(0,93)$ \\
\hline
\end{tabular}

$* p<0,05 ; * *<0,01$. Pearson Produkt-Moment-Korrelation (2-seitig). Interne Konsistenzen sind in Klammern in der Diagonalen angegeben

(FLMA, Richter und Wardanjan 2000) umfasst 24 Items (z.B. Ich kann beeinflussen, welche Arbeit mir zugeteilt wird, $M=76,26 ; S D=13,54 ; \alpha=0,93)$.

Die Befragten wurden gebeten die Selbstauskünfte auf einer 11-stufigen Antwortskala von $0=$,trifft überhaupt

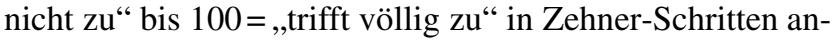
zugeben. Die internen Konsistenzen der Skalen sind als gut bis sehr gut zu bezeichnen. Während die Skaleninterkorrelationen zum Bruttojahresgehalt nur moderat ausfallen, sind die Zusammenhänge der Skalen untereinander im mittleren Bereich anzusiedeln (vgl. Tab. 1).

\subsection{Ergebnisse}

\subsubsection{Fragebogenkonstruktion}

Itemgenerierung Als Ausgangsbasis der Itemformulierung dienten neben dem Kasseler-Kompetenz-Raster (KKR; Kauffeld 2006a), verschiedene Kompetenzmodelle aus Unternehmen (vgl. Grote et al. 2012) sowie Definitionen der vier Facetten Fach-, Methoden-, Sozial- und Selbstkompetenz (vgl. Weinert 2001; Kauffeld 2006a; Sonntag und Schaper 1999). Jedes Item wurde von $0=$,trifft überhaupt

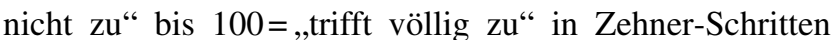
verankert. Neben positiven Items wurden in Anlehnung an das KRR für die Methoden-, Sozial- und Selbstkompetenz auch negative Items formuliert. Die negativ formulierten Items wurden für die Analysen invertiert. Nach Expertendiskussionen mit Forscherkollegen und Unternehmensvertretern resultierten für die Fach- 61 Items, die Methoden61 Items (davon 18 negativ formulierte Aussagen), die Sozial- 118 Items (davon 41 negativ formulierte Aussagen) und die Selbstkompetenz 93 Items (davon 44 negativ formulierte Aussagen). Neben der Einschätzung ihrer Kompetenz wurden die Befragten gebeten die Bedeutung der einzelnen Aspekte für Ihre Arbeit ebenfalls von $0=$,trifft

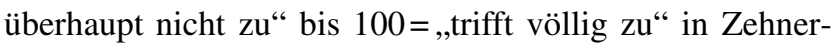
Schritten einzuschätzen. Die Abfolge der Items innerhalb der Kompetenzfacetten erfolgte zufällig.

Itemselektion Die Vorversion des KRI wurde mittels eines mehrstufigen Selektionsverfahrens in seine endgültige Struktur überführt. In die Endversion des Fragebogens wur- den diejenigen Items aufgenommen, die in der ersten Stichprobe die folgenden Kriterien erfüllten:

1. Verständlichkeit: Aussagen, die fünf oder mehr der 156 Befragten der ersten Stichprobe mit einem Fragezeichen kennzeichneten, wurden ausgeschlossen.

2. Bedeutung: Nur diejenigen Items, denen die Mitarbeiter auch eine Bedeutung für ihre Arbeit zuweisen, werden aufgenommen. Als cut-off Kriterium wurde der Wert 65 definiert.

Anschließend mussten die verbleibenden Items faktorenanalytischen Kriterien standhalten. Dabei wurden die vier Kompetenzfacetten separat faktorenanalytisch untersucht. Zur Durchführung der EFA wurde die Hauptkomponentenanalyse als Faktorextraktionsverfahrens gewählt. Um die resultierenden Faktoren inhaltlich sinnvoll interpretieren $\mathrm{zu}$ können, wurden die Faktoren anschließend orthogonal rotiert (Varimax-Kriterium; Kaiser 1958).

3. Eindeutigkeit der Faktorinterpretation: Items mit Faktorladungen $a<0,50$ (Konvention; Backhaus et al. 2000) und Items, die bei der Ladung auf mehreren Faktoren nicht in den Ladungshöhen die Distanz von mindestens $\Delta a=0,20$ aufweisen, wurden ausgeschlossen.

4. Skalenanalytische Kriterien: Jeder Faktor sollte aus mindestens drei Items bestehen. Aus Gründen der Ökonomie sollte eine Skala maximal fünf Items umfassen. Wenn nach den Kriterien mehr als fünf Items verblieben, wurden die Items mit den höchsten Ladungen präferiert (Positionierung des eigenen Standpunktes, Motivation von anderen, Rücksichtnahme und Besonnenheit, Mitwirkung, Verantwortungsübernahme und Initiative). Bei der Skala Verantwortungsübernahme und Initiative (Selbstkompetenz) wurden die Items mit den höchsten Ladungen mittels Korrelationsanalyse auf Redundanz geprüft und unter den redundanten die Items ausgewählt, die keine Fremdworte enthalten.

5. Itemanalytische Kriterien: Items, die eine Trennschärfe unter $r_{i t}=0,30$ aufwiesen, wurden ausgeschlossen (jeweils ein Item der Skalen Planung und Selbstmanagement).

Es resultieren 80 Items: 14 der Fach-, 24 der Methoden-, 24 der Sozial- und 18 der Selbstkompetenz. Ergebnisse der 
Tab. 2 EFA zur Faktorenstruktur der Fachkompetenz

\begin{tabular}{|c|c|c|c|c|c|c|c|c|c|c|}
\hline & 1 & 2 & 3 & 4 & M & SD & $\mathrm{R}_{\mathrm{it}}$ & $\mathrm{P}$ & ZS & $\mathrm{ZK}_{\mathrm{K}}$ \\
\hline \multicolumn{11}{|c|}{ Konzeptionelles Denken (4) 17,79\%; Innovatives Prozessdenken (10) 38,95\% } \\
\hline \multirow{2}{*}{$\begin{array}{l}\text { fk04/fk13-Es gelingt mir gut, mich vom konkreten Ein- } \\
\text { zelfall in meiner Arbeit zu lösen und alles von einer kon- } \\
\text { zeptionellen, übergeordneten Ebene aus zu betrachten }\end{array}$} & 0,77 & 0,18 & \multirow[t]{2}{*}{0,17} & \multirow[t]{2}{*}{0,17} & 66,46 & 20,47 & 0,71 & 67 & $-3,32 * *$ & $-0,19$ \\
\hline & $\mathbf{0 , 7 2}$ & 0,24 & & & 67,46 & 18,82 & 0,66 & 67 & $-3,15^{* *}$ & $-0,97$ \\
\hline \multirow{2}{*}{$\begin{array}{l}\text { fk07/fk23-Mich zeichnet aus, dass ich von vornherein } \\
\text { mögliche Stolpersteine bei der Entwicklung von Lösun- } \\
\text { gen bedenke }\end{array}$} & 0,71 & 0,11 & \multirow[t]{2}{*}{0,19} & \multirow[t]{2}{*}{0,33} & 68,75 & 17,66 & 0,70 & 69 & $-5,34 * *$ & $2,81 * *$ \\
\hline & 0,48 & 0,30 & & & 66,95 & 18,67 & 0,52 & 67 & $-4,07 * *$ & $-0,55$ \\
\hline \multirow{2}{*}{$\begin{array}{l}\text { fk08/fk24-Es gehört zu meinen Stärken, Lösungen nicht } \\
\text { nur für den Einzelfall bei meiner Arbeit zu erarbeiten, } \\
\text { sondern konzeptionell zu betrachten }\end{array}$} & 0,69 & - & \multirow[t]{2}{*}{0,19} & \multirow[t]{2}{*}{0,46} & 66,60 & 18,82 & 0,76 & 67 & $-4,52 * *$ & 1,51 \\
\hline & 0,78 & & & & 70,72 & 17,54 & 0,69 & 71 & $-3,03 * *$ & $-0,84$ \\
\hline \multirow{2}{*}{$\begin{array}{l}\text { fk02/fk05-Den Arbeitsprozess als Ganzes zu sehen, d.h. } \\
\text { in der gesamten Prozesskette zu denken, fällt mir leicht }\end{array}$} & $\mathbf{0 , 6 0}$ & 0,24 & \multirow[t]{2}{*}{0,23} & \multirow[t]{2}{*}{0,21} & 74,44 & 16,29 & 0,59 & 75 & $-3,12 * *$ & 0,45 \\
\hline & 0,69 & 0,29 & & & 75,64 & 15,04 & 0,61 & 76 & $-4,29 * *$ & 0,46 \\
\hline \multicolumn{11}{|l|}{ Kenntnis der Organisation und Abläufe (4) 16,17\% bzw. 14,23\% } \\
\hline \multirow{2}{*}{$\begin{array}{l}\text { fk13/fk51-Es fällt mir leicht zu sagen, wer für was in den } \\
\text { jeweiligen Fachgebieten zuständig bzw. Experte ist }\end{array}$} & 0,15 & 0,79 & \multirow[t]{2}{*}{-} & \multirow[t]{2}{*}{0,20} & 80,49 & 17,83 & 0,65 & 81 & $-4,49 * *$ & $-0,01$ \\
\hline & 0,31 & $\mathbf{0 , 7 0}$ & & & 77,40 & 17,85 & 0,51 & 77 & $-6,34 * *$ & 1,18 \\
\hline \multirow{2}{*}{$\begin{array}{l}\text { fk11/fk49-Das Ausführen von fachlichen Routinetätigkei- } \\
\text { ten fällt mir leicht }\end{array}$} & $-0,30$ & 0,71 & \multirow[t]{2}{*}{0,21} & \multirow[t]{2}{*}{-} & 85,14 & 16,43 & 0,39 & 85 & $-8,68 * *$ & $8,50 * *$ \\
\hline & $-0,33$ & 0,71 & & & 75,66 & 24,02 & 0,16 & 76 & $-8,30 * *$ & 1,84 \\
\hline \multirow{2}{*}{$\begin{array}{l}\text { fk14/fkfk53-Mein fachliches Wissen sinnvoll einzusetzen } \\
\text { fällt mir leicht }\end{array}$} & 0,25 & 0,69 & \multirow[t]{2}{*}{0,24} & \multirow[t]{2}{*}{0,18} & 82,92 & 13,53 & 0,56 & 83 & $-4,63 * *$ & $3,44 * *$ \\
\hline & 0,36 & 0,65 & & & 80,69 & 14,98 & 0,46 & 81 & $-6,80^{* *}$ & 2,44 \\
\hline \multirow{2}{*}{$\begin{array}{l}\text { fk09/fk36-Ich kenne den Aufbau der Organisation (Orga- } \\
\text { nigramm) }\end{array}$} & 0,33 & 0,61 & \multirow[t]{2}{*}{-} & \multirow[t]{2}{*}{0,12} & 81,46 & 19,96 & 0,51 & 82 & $-7,27 * *$ & $5,93 * *$ \\
\hline & 0,44 & 0,44 & & & 80,56 & 20,52 & 0,33 & 81 & $-7,94 * *$ & 1,31 \\
\hline \multicolumn{11}{|l|}{ Problemerkennung $^{\mathrm{a}}$ (3) $14,09 \%$} \\
\hline fk03/fk06-Abläufe und Prozesse in der Organisation in & $\mathbf{0 , 7 1}$ & 0,13 & 0,73 & 0,17 & 68,39 & 20,08 & 0,56 & 68 & $-2,10$ & $-1,25$ \\
\hline Frage zu stellen fällt mir leicht & & & & & 71,63 & 18,42 & 0,64 & 72 & $-2,91 * *$ & $-2,54$ \\
\hline fk01/fk01-Probleme und Veränderungsbedarf in meinem & 0,23 & 0,15 & 0,67 & 0,23 & 75,23 & 13,45 & 0,39 & 75 & $-2,58 * *$ & 0,54 \\
\hline Fachgebiet zu erkennen fällt mir leicht & 0,66 & 0,21 & & & 74,57 & 14,87 & 0,53 & 75 & $-5,31 * *$ & $4,08 * *$ \\
\hline fk12/fk04-,,Altbewährtes“ bzw. Dinge in meinem Fachge- & 0,23 & 0,23 & 0,58 & 0,27 & 66,09 & 19,63 & 0,56 & 66 & $-0,96$ & $-1,47$ \\
\hline $\begin{array}{l}\text { biet, die schon lange Bestand haben, kritisch zu hinterfra- } \\
\text { gen ist eine meiner Stärken }\end{array}$ & 0,60 & & & & 70,96 & 17,96 & 0,55 & 71 & $-4,06 * *$ & $-0,14$ \\
\hline Entwicklung von Lösungen ${ }^{\mathrm{a}}$ (3) 13,19\% & & & & & & & & & & \\
\hline fk05/fk17-Es ist eine meiner Stärken, neue Ideen in mei- & 0,19 & - & 0,23 & $\mathbf{0 , 8 2}$ & 66,53 & 22,01 & 0,72 & 67 & $-2,84 * *$ & $-0,70$ \\
\hline nem Fachgebiet zu entwickeln & $\mathbf{0 , 8 1}$ & & & & 69,56 & 19,51 & 0,77 & 70 & $-5,07 * *$ & 1,00 \\
\hline fk10/fk41-Neue Ideen zur Verbesserung meiner Arbeit zu & 0,17 & 0,28 & 0,19 & 0,79 & 69,24 & 18,51 & 0,74 & 70 & $-2,74 * *$ & $-0,59$ \\
\hline entwickeln ist eine meiner Stärken & $\mathbf{0 , 7 3}$ & 0,14 & & & 72,11 & 17,45 & 0,65 & 72 & $-4,11 * *$ & 0,05 \\
\hline fk06/fk21-Es ist eine meiner Stärken, verschiedene fach- & 0,30 & 0,21 & 0,36 & 0,63 & 71,32 & 16,69 & 0,60 & 72 & $-2,67 * *$ & $-0,85$ \\
\hline $\begin{array}{l}\text { liche Ideen in meinem Fachgebiet miteinander zu verbin- } \\
\text { den }\end{array}$ & $\mathbf{0 , 8 0}$ & & & & 70,94 & 16,87 & 0,69 & 71 & $-4,15 * *$ & 2,07 \\
\hline
\end{tabular}

Itemnummer im KRI/Itemnummer in der Vorversion des KRI. Der obere/erste Wert stellt die Ergebnisse in der ersten Stichprobe $(N=144)$, der untere/zweite Wert die Ergebnisse in der zweiten Stichprobe $(N=349)$ dar. Angegeben werden die Varimax-rotierten Faktorladungen der Items und ihre Zuordnung zu den Faktoren. Fett gedruckt sind die Ladungen, die für die Faktorinterpretation herangezogen wurden. Ladungen von $a<0,10$ sind nicht ausgewiesen

${ }^{a}$ Teilaspekte der Skala Innovatives Prozessdenken in der zweiten Stichprobe. In Klammern hinter den Skalenüberschriften steht die Anzahl der Items, dahinter wird die erklärte Varianz nach Rotation für den Faktor angegeben. Die Rotation ist in 6 bzw. 3 Iterationen konvergiert

vier Faktoren- sowie der Itemanalysen beider Stichproben sind in Tab. 2, 3, 4, 5, 6 und 7 dargestellt.

\section{Skalen des KRI}

Fachkompetenz Innerhalb der Fachkompetenz konnten in der ersten Stichprobe die vier Faktoren Problemerkennung und Entwicklung von Lösungen, Konzeptionelles Denken, Kenntnis der Organisation und Abläufe extrahiert werden, die zusammen $61 \%$ der Varianz erklären (vgl. Tab. 2). Die
Faktoren lassen sich in der zweiten Stichprobe teilweise replizieren. In der zweiten Stichprobe führt nur eine ZweiFaktoren-Lösung zu sinnvoll interpretierbaren Ergebnissen, die $53 \%$ der Varianz erklärt. Inhaltlich umfasst der Faktor Innovatives Prozessdenken, die Skalen Konzeptionelles Denken, Problemerkennung und Entwicklung von Lösungen der ersten Stichprobe. Die Skala Kenntnis der Organisation und Abläufe wurde weitgehend - bis auf das Item fk09Ich kenne den Aufbau der Organisation (Organigramm), das auf beide Faktoren mit gleich hohen Ladungen lädt - bestätigt. $\mathrm{Zu}$ vermuten ist, dass die drei Skalen Konzeptionelles 
Tab. 3 EFA zur Faktorenstruktur der Methodenkompetenz

\begin{tabular}{|c|c|c|c|c|c|c|c|c|c|c|c|c|}
\hline & 1 & 2 & 3 & 4 & 5 & 6 & M & SD & $\mathrm{R}_{\mathrm{it}}$ & $\mathrm{P}$ & Zs & ZK \\
\hline \multicolumn{13}{|c|}{ Moderation und Präsentation (5) 15,32\% bzw. 14,23\% } \\
\hline $\begin{array}{l}\text { mkp18/mkp39-Es gehört zu meinen Stär- } \\
\text { ken, schwierige Sachverhalte in meinem } \\
\text { Arbeitsbereich für andere (z. B. Kollegen } \\
\text { und Vorgesetzte) verständlich zu erläutern }\end{array}$ & $\begin{array}{l}\mathbf{0 , 8 3} \\
\mathbf{0 , 8 3}\end{array}$ & - & 0,10 & - & $-0,12$ & $-0,13$ & $\begin{array}{l}71,01 \\
70,86\end{array}$ & $\begin{array}{l}20,38 \\
19,15\end{array}$ & $\begin{array}{l}0,75 \\
0,72\end{array}$ & $\begin{array}{l}71 \\
71\end{array}$ & $\begin{array}{l}-5,34 * * \\
-4,39 * *\end{array}$ & $\begin{array}{l}1,86 \\
-0,66\end{array}$ \\
\hline $\begin{array}{l}\text { mkp19/mkp40-Es gelingt mir gut, Vorge- } \\
\text { hensweisen oder Ergebnisse auch für andere } \\
\text { nachvollziehbar zu dokumentieren }\end{array}$ & $\begin{array}{l}\mathbf{0 , 7 5} \\
\mathbf{0 , 7 7}\end{array}$ & $\begin{array}{l}0,18 \\
0,22\end{array}$ & 0,25 & - & $-0,11$ & 0,20 & $\begin{array}{l}71,01 \\
71,69\end{array}$ & $\begin{array}{l}18,11 \\
18,68\end{array}$ & $\begin{array}{l}0,75 \\
0,64\end{array}$ & $\begin{array}{l}70 \\
72\end{array}$ & $\begin{array}{l}-3,43 * * \\
-7,23 * *\end{array}$ & $\begin{array}{l}0,42 \\
3,77 * *\end{array}$ \\
\hline $\begin{array}{l}\text { mkp11/mkp03-Es ist eine meiner Stärken, } \\
\text { arbeitsbezogene Sachverhalte klar und deut- } \\
\text { lich darzustellen }\end{array}$ & $\begin{array}{l}0,71 \\
0,71\end{array}$ & $\begin{array}{l}0,32 \\
0,32\end{array}$ & $\begin{array}{l}0,20 \\
0,20\end{array}$ & 0,18 & $-0,14$ & - & $\begin{array}{l}75,81 \\
71,88\end{array}$ & $\begin{array}{l}16,43 \\
16,96\end{array}$ & $\begin{array}{l}0,67 \\
0,71\end{array}$ & $\begin{array}{l}75 \\
72\end{array}$ & $\begin{array}{l}-3,88 * * \\
-3,95 * *\end{array}$ & $\begin{array}{l}0,97 \\
-0,10\end{array}$ \\
\hline $\begin{array}{l}\text { mkp17/mkp34-Es ist eine meiner Stärken, } \\
\text { Gruppen bzw. Workshops zu bestimmten } \\
\text { Fragestellungen zu moderieren }\end{array}$ & $\begin{array}{l}0,69 \\
0,69\end{array}$ & $-0,20$ & $\begin{array}{l}0,22 \\
0,28\end{array}$ & 0,10 & 0,15 & $\begin{array}{l}0,31 \\
-0,26\end{array}$ & $\begin{array}{l}58,91 \\
61,62\end{array}$ & $\begin{array}{l}25,87 \\
23,90\end{array}$ & $\begin{array}{l}0,54 \\
0,59\end{array}$ & $\begin{array}{l}59 \\
62\end{array}$ & $\begin{array}{l}-2,91 * * \\
-2,92 * *\end{array}$ & $\begin{array}{l}-0,56 \\
-1,92\end{array}$ \\
\hline $\begin{array}{l}\text { mkp12/mkp05-Es ist eine meiner Stärken, } \\
\text { arbeitsbezogene (Zwischen-) Ergebnisse } \\
\text { zusammenzufassen }\end{array}$ & $\begin{array}{l}\mathbf{0 , 5 8} \\
\mathbf{0 , 6 8}\end{array}$ & $\begin{array}{l}0,31 \\
0,34\end{array}$ & $\begin{array}{l}0,35 \\
0,25\end{array}$ & $\begin{array}{l}0,16 \\
0,16\end{array}$ & $-0,19$ & - & $\begin{array}{l}72,40 \\
68,35\end{array}$ & $\begin{array}{l}17,17 \\
18,14\end{array}$ & $\begin{array}{l}0,56 \\
0,71\end{array}$ & $\begin{array}{l}71 \\
68\end{array}$ & $\begin{array}{l}-4,55 * * \\
-4,48 * *\end{array}$ & $\begin{array}{l}2,80^{* *} \\
0,82\end{array}$ \\
\hline \multicolumn{13}{|l|}{ Planung (4) $14,51 \%$ bzw. $14,92 \%$} \\
\hline $\begin{array}{l}\text { mkp03/mkp11-Es fällt mir leicht, bei meiner } \\
\text { Arbeit Prioritäten zu setzen }\end{array}$ & 0,12 & $\begin{array}{l}\mathbf{0 , 7 9} \\
\mathbf{0 , 7 4}\end{array}$ & $\begin{array}{l}0,18 \\
0,20\end{array}$ & 0,10 & $-0,21$ & 0,20 & $\begin{array}{l}79,30 \\
73,95\end{array}$ & $\begin{array}{l}15,16 \\
17,77\end{array}$ & $\begin{array}{l}0,74 \\
0,68\end{array}$ & $\begin{array}{l}79 \\
74\end{array}$ & $\begin{array}{l}-3,94 * * \\
-4,13 * *\end{array}$ & $\begin{array}{l}0,39 \\
-0,46\end{array}$ \\
\hline $\begin{array}{l}\text { mkp02/mkp08-Es gehört zu meinen Stärken, } \\
\text { für umfangreiche fachliche Tätigkeiten die } \\
\text { erforderliche Zeit einzuplanen }\end{array}$ & 0,11 & $\begin{array}{l}0,74 \\
0,79\end{array}$ & 0,13 & - & $-0,14$ & 0,11 & $\begin{array}{l}72,40 \\
67,34\end{array}$ & $\begin{array}{l}18,99 \\
19,77\end{array}$ & $\begin{array}{l}0,56 \\
0,66\end{array}$ & $\begin{array}{l}71 \\
67\end{array}$ & $\begin{array}{l}-4,57 * * \\
-4,29 * *\end{array}$ & $\begin{array}{l}1,85 \\
0,59\end{array}$ \\
\hline $\begin{array}{l}\text { mkp01/mkp17-Zielgerichtet bei meiner } \\
\text { Arbeit zu handeln fällt mir leicht }\end{array}$ & $\begin{array}{l}0,19 \\
0,22\end{array}$ & $\begin{array}{l}\mathbf{0 , 6 9} \\
\mathbf{0 , 7 3}\end{array}$ & $\begin{array}{l}0,32 \\
0,12\end{array}$ & 0,26 & $\begin{array}{l}-0,23 \\
-0,21\end{array}$ & - & $\begin{array}{l}79,77 \\
75,52\end{array}$ & $\begin{array}{l}15,18 \\
15,21\end{array}$ & $\begin{array}{l}0,73 \\
0,69\end{array}$ & $\begin{array}{l}79 \\
76\end{array}$ & $\begin{array}{l}-5,05^{* *} \\
-4,60^{* *}\end{array}$ & $\begin{array}{l}2,80 * * \\
2,02\end{array}$ \\
\hline $\begin{array}{l}\text { mkp08/mkp16-Das Koordinieren von ein- } \\
\text { zelnen Arbeitsschritten fällt mir leicht }\end{array}$ & $\begin{array}{l}0,32 \\
0,26\end{array}$ & $\begin{array}{l}\mathbf{0 , 6 1} \\
\mathbf{0 , 7 1}\end{array}$ & $\begin{array}{l}0,24 \\
0,21\end{array}$ & $\begin{array}{l}0,23 \\
0,16\end{array}$ & $-0,11$ & $\begin{array}{l}0,25 \\
-0,11\end{array}$ & $\begin{array}{l}77,98 \\
75,49\end{array}$ & $\begin{array}{l}14,38 \\
14,51\end{array}$ & $\begin{array}{l}0,69 \\
0,63\end{array}$ & $\begin{array}{l}78 \\
75\end{array}$ & $\begin{array}{l}-4,28 * * \\
-4,07 * *\end{array}$ & $\begin{array}{l}2,30 \\
1,19\end{array}$ \\
\hline Reflexivität (4) 11,04\% bzw. 9,77\% & & & & & & & & & & & & \\
\hline $\begin{array}{l}\text { mkp09/mkp21-Es fällt mir leicht, nach Ab- } \\
\text { schluss eines Projektes oder eines größeren } \\
\text { Arbeitsschrittes systematisch zu untersu- } \\
\text { chen, was gut und was schlecht gelaufen } \\
\text { ist }\end{array}$ & $\begin{array}{l}0,19 \\
0,33\end{array}$ & $\begin{array}{l}0,27 \\
0,42\end{array}$ & $\begin{array}{l}0,79 \\
0,63\end{array}$ & - & - & 0,15 & $\begin{array}{l}71,40 \\
68,34\end{array}$ & $\begin{array}{l}19,19 \\
19,06\end{array}$ & $\begin{array}{l}0,74 \\
0,67\end{array}$ & $\begin{array}{l}70 \\
68\end{array}$ & $\begin{array}{l}-5,38 * * \\
-3,72 * *\end{array}$ & $\begin{array}{l}3,41 * * \\
-0,49\end{array}$ \\
\hline $\begin{array}{l}\text { mkp10/mkp22-Es gehört zu meinen Stär- } \\
\text { ken, nach einer abgeschlossenen Arbeit das } \\
\text { Ergebnis mit meinen Erwartungen abzuglei- } \\
\text { chen }\end{array}$ & $\begin{array}{l}0,31 \\
0,26\end{array}$ & $\begin{array}{l}0,25 \\
0,39\end{array}$ & $\begin{array}{l}\mathbf{0 , 7 7} \\
\mathbf{0 , 7 0}\end{array}$ & - & - & 0,10 & $\begin{array}{l}72,40 \\
68,11\end{array}$ & $\begin{array}{l}19,95 \\
18,96\end{array}$ & $\begin{array}{l}0,83 \\
0,70\end{array}$ & $\begin{array}{l}72 \\
68\end{array}$ & $\begin{array}{l}-4,48 * * \\
-3,61 * *\end{array}$ & $\begin{array}{l}2,55 \\
-0,23\end{array}$ \\
\hline $\begin{array}{l}\text { mkp16/mkp24-Es fällt mir leicht, Ergebnis- } \\
\text { se meiner Arbeit zu bewerten }\end{array}$ & $\begin{array}{l}0,34 \\
0,25\end{array}$ & 0,20 & $\begin{array}{l}\mathbf{0 , 7 0} \\
\mathbf{0 , 6 7}\end{array}$ & $\begin{array}{l}0,20 \\
0,18\end{array}$ & - & $\begin{array}{l}0,10 \\
-0,13\end{array}$ & $\begin{array}{l}73,95 \\
71,24\end{array}$ & $\begin{array}{l}15,68 \\
17,73\end{array}$ & $\begin{array}{l}0,61 \\
0,58\end{array}$ & $\begin{array}{l}73 \\
71\end{array}$ & $\begin{array}{l}-2,35 \\
-7,22 * *\end{array}$ & $\begin{array}{l}0,35 \\
5,17 * *\end{array}$ \\
\hline $\begin{array}{l}\text { mkp15/mkp23-Es zeichnet mich aus, im } \\
\text { Nachhinein zu überlegen, wie ich Schwie- } \\
\text { rigkeiten und Probleme beim nächsten Mal } \\
\text { besser lösen kann }\end{array}$ & $\begin{array}{l}0,33 \\
0,11\end{array}$ & 0,41 & $\begin{array}{l}\mathbf{0 , 6 8} \\
0,73\end{array}$ & 0,25 & $\begin{array}{l}0,14 \\
-0,12\end{array}$ & $-0,13$ & $\begin{array}{l}75,97 \\
72,23\end{array}$ & $\begin{array}{l}17,48 \\
16,18\end{array}$ & $\begin{array}{l}0,78 \\
0,52\end{array}$ & $\begin{array}{l}75 \\
72\end{array}$ & $\begin{array}{l}-4,45 * * \\
-4,61 * *\end{array}$ & $\begin{array}{l}1,89 \\
0,57\end{array}$ \\
\hline
\end{tabular}

Itemnummer im KRI/Itemnummer in der Vorversion des KRI. (i) steht für negativ gepoltes Item. Der obere/erste Wert stellt die Ergebnisse in der ersten Stichprobe $(N=129)$, der untere/zweite Wert die Ergebnisse in der zweiten Stichprobe $(N=343)$ dar. Angegeben werden die Varimaxrotierten Faktorladungen der Items und ihre Zuordnung zu den Faktoren. Fett gedruckt sind die Ladungen, die für die Faktorinterpretation herangezogen wurden. Ladungen von $a<0,10$ sind nicht ausgewiesen. In Klammern hinter den Skalenüberschriften steht die Anzahl der Items, dahinter wird die erklärte Varianz nach Rotation für den Faktor angegeben. Die Rotation ist in 10 bzw. 8 Iterationen konvergiert

Denken, Problemerkennung und Entwicklung von Lösungen Teilaspekte der übergreifenden Dimension Innovatives Prozessdenken abbilden. Da das Ziel der Fragebogenentwicklung u.a. darin bestand, möglichst detaillierte Informationen über die Ausprägungen bzgl. der einzelnen Kompetenzfacetten zu erheben, wird die Vier-Faktoren-Struktur beibehalten.
Methodenkompetenz Für die Methodenkompetenz konnten in der ersten Stichprobe die sechs Faktoren Moderation und Präsentation, Planung, Reflexivität, Umgang mit IKT und Technik, Konzentration und Aufgabenverteilung ermittelt werden, die in der zweiten Stichprobe weitgehend bestätigt werden konnten. Lediglich 2 von 24 Items laden auf einem anderen Faktor am höchsten. Die extrahierten Fak- 
Tab. 4 (Fortsetzung): EFA zur Faktorenstruktur der Methodenkompetenz

\begin{tabular}{|c|c|c|c|c|c|c|c|c|c|c|c|c|}
\hline & 1 & 2 & 3 & 4 & 5 & 6 & $\mathrm{M}$ & SD & $\mathrm{R}_{\mathrm{it}}$ & $\mathrm{P}$ & ZS & $\mathrm{ZK}_{\mathrm{K}}$ \\
\hline \multicolumn{13}{|c|}{ Umgang mit IKT und Technik (4) 10,06\% bzw. 13,43\% } \\
\hline $\begin{array}{l}\text { mkp04/mkp41-Der Umgang mit PC und } \\
\text { Software fällt mir leicht }\end{array}$ & 0,13 & - & - & $\begin{array}{l}\mathbf{0 , 9 3} \\
\mathbf{0 , 9 2}\end{array}$ & - & - & $\begin{array}{l}79,38 \\
78,48\end{array}$ & $\begin{array}{l}20,61 \\
22,93\end{array}$ & $\begin{array}{l}0,85 \\
0,86\end{array}$ & $\begin{array}{l}77 \\
78\end{array}$ & $\begin{array}{l}-5,94 * * \\
-9,38 * *\end{array}$ & $\begin{array}{l}3,07 * * \\
3,53 * *\end{array}$ \\
\hline $\begin{array}{l}\text { mkp13/mkp42-Der Umgang mit Online- } \\
\text { diensten und dem Internet fällt mir leicht }\end{array}$ & $\begin{array}{l}0,11 \\
0,17\end{array}$ & $-0,10$ & 0,11 & $\begin{array}{l}\mathbf{0 , 9 1} \\
\mathbf{0 , 8 6}\end{array}$ & - & - & $\begin{array}{l}74,26 \\
77,65\end{array}$ & $\begin{array}{l}26,71 \\
23,52\end{array}$ & $\begin{array}{l}0,81 \\
0,77\end{array}$ & $\begin{array}{l}73 \\
78\end{array}$ & $\begin{array}{l}-6,10 * * \\
-9,54 * *\end{array}$ & $\begin{array}{l}1,62 \\
4,02 * *\end{array}$ \\
\hline $\begin{array}{l}\text { mkp05/fk31-Der Umgang mit Arbeitshilfs- } \\
\text { mitteln /Maschinen (auch PC etc.) fällt mir } \\
\text { leicht }\end{array}$ & 0,14 & $\begin{array}{l}0,28 \\
0,14\end{array}$ & 0,13 & $\begin{array}{l}\mathbf{0 , 7 8} \\
\mathbf{0 , 8 9}\end{array}$ & - & - & $\begin{array}{l}82,33 \\
80,55\end{array}$ & $\begin{array}{l}16,84 \\
20,38\end{array}$ & $\begin{array}{l}0,68 \\
0,84\end{array}$ & $\begin{array}{l}81 \\
81\end{array}$ & $\begin{array}{l}-6,80 * * \\
-10,75 * *\end{array}$ & $\begin{array}{l}5,28 * * \\
7,60 * *\end{array}$ \\
\hline $\begin{array}{l}\text { mkp14/mkp43-Der Umgang mit Datenbank- } \\
\text { systemen (z.B. SAP) fällt mir leicht }\end{array}$ & 0,15 & 0,15 & 0,13 & $\begin{array}{l}0,72 \\
0,75\end{array}$ & - & 0,13 & $\begin{array}{l}58,29 \\
64,32\end{array}$ & $\begin{array}{l}32,24 \\
28,80\end{array}$ & $\begin{array}{l}0,61 \\
0,64\end{array}$ & $\begin{array}{l}57 \\
64\end{array}$ & $\begin{array}{l}-2,34 \\
-5,51 * *\end{array}$ & $\begin{array}{l}-2,58 * * \\
-1,78\end{array}$ \\
\hline \multicolumn{13}{|l|}{ Konzentration (4) 7,79\% bzw. 9,87\% } \\
\hline $\begin{array}{l}\text { mkn03/mkn03-Mich in Details und Bei- } \\
\text { spielen (Kleinigkeiten) in meiner Arbeit zu } \\
\text { verlieren, passiert mir leicht. (i) }\end{array}$ & - & $-0,17$ & - & - & $\begin{array}{l}\mathbf{0 , 8 3} \\
\mathbf{0 , 7 7}\end{array}$ & $\begin{array}{l}-0,18 \\
0,31\end{array}$ & $\begin{array}{l}27,96 \\
37,27\end{array}$ & $\begin{array}{l}21,29 \\
25,92\end{array}$ & $\begin{array}{l}0,75 \\
0,67\end{array}$ & $\begin{array}{l}72 \\
63\end{array}$ & $\begin{array}{l}-5,11 * * \\
-3,59 * *\end{array}$ & $\begin{array}{l}1,71 \\
-2,78 * *\end{array}$ \\
\hline $\begin{array}{l}\text { mkn02/mkn02-Ich neige dazu, etwas anzu- } \\
\text { fangen und nicht zu Ende zu bringen. (i) }\end{array}$ & $-0,14$ & $\begin{array}{l}-0,35 \\
-0,15\end{array}$ & $-0,13$ & - & $\begin{array}{l}\mathbf{0 , 7 3} \\
\mathbf{0 , 8 1}\end{array}$ & - & $\begin{array}{l}22,89 \\
32,31\end{array}$ & $\begin{array}{l}22,37 \\
26,32\end{array}$ & $\begin{array}{l}0,66 \\
0,55\end{array}$ & $\begin{array}{l}77 \\
68\end{array}$ & $\begin{array}{l}-7,01 * * \\
-6,13 * *\end{array}$ & $\begin{array}{l}4,03 * * \\
-1,64\end{array}$ \\
\hline $\begin{array}{l}\text { mkn04/mkn04-Ich neige dazu, in meinem } \\
\text { Arbeitsbereich pedantisch zu sein. (i) }\end{array}$ & $-0,11$ & $\begin{array}{l}0,16 \\
0,22\end{array}$ & - & $\begin{array}{l}-0,26 \\
-0,14\end{array}$ & $\begin{array}{l}\mathbf{0 , 6 1} \\
\mathbf{0 , 4 1}\end{array}$ & $\begin{array}{l}-0,13 \\
0,55\end{array}$ & $\begin{array}{l}37,06 \\
40,64\end{array}$ & $\begin{array}{l}29,24 \\
29,03\end{array}$ & $\begin{array}{l}0,31 \\
0,35\end{array}$ & $\begin{array}{l}63 \\
59\end{array}$ & $\begin{array}{l}-1,98 \\
-2,69 * *\end{array}$ & $\begin{array}{l}-2,79 * * \\
-4,26 * *\end{array}$ \\
\hline $\begin{array}{l}\text { mkn01/mkn01-Es passiert mir leicht, dass } \\
\text { ich zwischen Aufgaben springe und damit } \\
\text { nicht zum Wesentlichen komme. (i) }\end{array}$ & $-0,13$ & $\begin{array}{l}-0,26 \\
-0,26\end{array}$ & - & - & $\begin{array}{l}\mathbf{0 , 5 9} \\
\mathbf{0 , 8 1}\end{array}$ & 0,12 & $\begin{array}{l}37,60 \\
44,64\end{array}$ & $\begin{array}{l}23,88 \\
25,00\end{array}$ & $\begin{array}{l}0,54 \\
0,60\end{array}$ & $\begin{array}{l}62 \\
55\end{array}$ & $\begin{array}{l}-2,39 \\
-1,27\end{array}$ & $\begin{array}{l}-1,99 \\
-3,85^{* * *}\end{array}$ \\
\hline Aufgabenverteilung (3) 6,83\% bzw. 6,90\% & & & & & & & & & & & & \\
\hline $\begin{array}{l}\text { mkn05/mkn13-Mir fällt es schwer, Aufga- } \\
\text { ben zu delegieren bzw. an andere abzuge- } \\
\text { ben. (i) }\end{array}$ & - & $-0,11$ & $-0,11$ & - & $\begin{array}{l}0,25 \\
0,31\end{array}$ & $\begin{array}{l}-\mathbf{0 , 8 1} \\
\mathbf{0 , 7 7}\end{array}$ & $\begin{array}{l}30,78 \\
39,20\end{array}$ & $\begin{array}{l}24,86 \\
27,13\end{array}$ & $\begin{array}{l}0,54 \\
0,41\end{array}$ & $\begin{array}{l}69 \\
61\end{array}$ & $\begin{array}{l}-2,43 \\
-2,66 * *\end{array}$ & $\begin{array}{l}-2,33 \\
-3,81 * *\end{array}$ \\
\hline $\begin{array}{l}\text { mkp06/mkp13-Es gehört zu meinen Stärken, } \\
\text { Aufgaben angemessen zu delegieren bzw. an } \\
\text { Mitarbeiter oder Kollegen zu verteilen }\end{array}$ & $\begin{array}{l}0,23 \\
0,20\end{array}$ & $\begin{array}{l}0,26 \\
0,49\end{array}$ & 0,18 & - & - & $\begin{array}{l}0,78 \\
-0,67\end{array}$ & $\begin{array}{l}67,29 \\
67,98\end{array}$ & $\begin{array}{l}21,90 \\
21,00\end{array}$ & $\begin{array}{l}0,75 \\
0,59\end{array}$ & $\begin{array}{l}67 \\
68\end{array}$ & $\begin{array}{l}-3,81 * * \\
-5,67 * *\end{array}$ & $\begin{array}{l}0,24 \\
1,06\end{array}$ \\
\hline $\begin{array}{l}\text { mkp07/mkp14-Einzelne Arbeitsschritte für } \\
\text { mich und andere zu konkretisieren fällt mir }\end{array}$ & $\begin{array}{l}0,29 \\
0,42\end{array}$ & $\begin{array}{l}0,30 \\
0,58\end{array}$ & $\begin{array}{l}0,27 \\
0,29\end{array}$ & 0,16 & - & $\begin{array}{l}0,51 \\
-0,23\end{array}$ & $\begin{array}{l}71,92 \\
73,40\end{array}$ & $\begin{array}{l}16,97 \\
16,23\end{array}$ & $\begin{array}{l}0,56 \\
0,36\end{array}$ & $\begin{array}{l}72 \\
73\end{array}$ & $\begin{array}{l}-5,75 * * \\
-5,44 * *\end{array}$ & $\begin{array}{l}4,60 * * \\
1,68\end{array}$ \\
\hline
\end{tabular}

leicht

Itemnummer im KRI/Itemnummer in der Vorversion des KRI. (i) steht für negativ gepoltes Item. Der obere/erste Wert stellt die Ergebnisse in der ersten Stichprobe $(N=129)$, der untere/zweite Wert die Ergebnisse in der zweiten Stichprobe $(N=343)$ dar. Angegeben werden die Varimaxrotierten Faktorladungen der Items und ihre Zuordnung zu den Faktoren. Fett gedruckt sind die Ladungen, die für die Faktorinterpretation herangezogen wurden. Ladungen von $a<0,10$ sind nicht ausgewiesen. In Klammern hinter den Skalenüberschriften steht die Anzahl der Items, dahinter wird die erklärte Varianz nach Rotation für den Faktor angegeben. Die Rotation ist in 10 bzw. 8 Iterationen konvergiert

toren erklären $66 \%$ der Varianz in der ersten und $69 \%$ in der zweiten Stichprobe (vgl. Tab. 3 und 4).

Sozialkompetenz Innerhalb der Sozialkompetenz können in der ersten Stichprobe die fünf Skalen Positionierung des eigenen Standpunktes, Motivation von Anderen, Akzeptanz und Anerkennung, Kontaktaufbau und -pflege sowie Rücksichtnahme und Besonnenheit unterschieden werden, die in der zweiten weitgehend Stichprobe bestätigt werden (Ausnahme 2 von 24 Items, die auf jeweils 2 Faktoren substantiell laden). Die fünf Faktoren erklären $62 \%$ der Varianz in der ersten Stichprobe und $69 \%$ der Varianz in der zweiten Stichprobe (vgl. Tab. 5 und 6).

Selbstkompetenz Innerhalb der Selbstkompetenz können in der ersten Stichprobe die vier Dimensionen Mitwirkung, Verantwortungsübernahme und Initiative, Veränderungsin- teresse sowie Selbstmanagement ermittelt werden, die sich in der zweiten Stichprobe weitgehend bestätigen lassen (Ausnahme 2 von 18 Items). Die extrahierten Faktoren erklären $60 \%$ Varianz in der ersten und $63 \%$ Varianz in der zweiten Stichprobe (vgl. Tab. 7).

Itemkennwerte Die Trennschärfen variieren in der ersten Stichprobe von $r_{i t}=0,31$ (Item mkn04, Skala Konzentration, fk11, Skala Kenntnis der Organisation und Abläufe) bis $r_{i t}=0,87$ (Item sop05, Skala Motivation von Anderen), in der zweiten Stichprobe von $r_{i t}=0,16$ (Item fk11, Skala Kenntnis der Organisation und Abläufe) bis $r_{i t}=0,86$ (Item mkp04, Skala Umgang mit IKT und Technik). In der ersten Stichprobe sind 74 Items als hoch, sechs Items als mittel trennscharf, in der zweiten Stichprobe 70 Items als hoch, neun als mittel und ein Item (fk11-Das Ausführen von fachlichen Routinetätigkeiten fällt mir leicht.) als gering trenn- 
Tab. 5 EFA zur Faktorenstruktur der Sozialkompetenz

\begin{tabular}{|c|c|c|c|c|c|c|c|c|c|c|c|c|}
\hline & 1 & 2 & 3 & 4 & 5 & 6 & $M$ & $S D$ & $R_{i t}$ & $P$ & $z s$ & $z \mathrm{~K}$ \\
\hline \multicolumn{13}{|c|}{ Positionierung des eigenen Standpunktes (5) 14,86\% bzw. 9,74\% } \\
\hline $\begin{array}{l}\text { sop09/sop34-Sachliche Kritik (gegenüber } \\
\text { meinen Kollegen, Mitarbeitern, Vorgesetz- } \\
\text { ten etc.) zu üben ist eine meiner Stärken }\end{array}$ & $\begin{array}{l}\mathbf{0 , 8 3} \\
\mathbf{0 , 6 5}\end{array}$ & $\begin{array}{l}0,20 \\
0,42\end{array}$ & $\begin{array}{l}0,21 \\
0,33\end{array}$ & - & - & - & $\begin{array}{l}66,64 \\
65,07\end{array}$ & $\begin{array}{l}21,14 \\
17,96\end{array}$ & $\begin{array}{l}0,79 \\
0,66\end{array}$ & $\begin{array}{l}67 \\
65\end{array}$ & $\begin{array}{l}-2,85 * * \\
-4,28 * *\end{array}$ & $\begin{array}{l}-1,16 \\
0,38\end{array}$ \\
\hline $\begin{array}{l}\text { sop19/sop68-Es fällt mir leicht, anderen zu } \\
\text { widersprechen }\end{array}$ & $\begin{array}{l}\mathbf{0 , 7 7} \\
\mathbf{0 , 7 6}\end{array}$ & $\begin{array}{l}0,22 \\
0,17\end{array}$ & - & $\begin{array}{l}0,30 \\
0,26\end{array}$ & 0,16 & - & $\begin{array}{l}71,79 \\
68,27\end{array}$ & $\begin{array}{l}18,90 \\
20,13\end{array}$ & $\begin{array}{l}0,73 \\
0,63\end{array}$ & $\begin{array}{l}72 \\
68\end{array}$ & $\begin{array}{l}-3,07 * * \\
-3,36 * *\end{array}$ & $\begin{array}{l}-0,41 \\
-1,87\end{array}$ \\
\hline $\begin{array}{l}\text { sop02/sop33-Die eigene Meinung (gegen- } \\
\text { über Kollegen, Mitarbeitern etc.) zu vertre- } \\
\text { ten fällt mir leicht }\end{array}$ & $\begin{array}{l}0,76 \\
0,60\end{array}$ & 0,15 & 0,14 & $\begin{array}{l}0,32 \\
0,57\end{array}$ & - & - & $\begin{array}{l}79,43 \\
76,59\end{array}$ & $\begin{array}{l}15,54 \\
18,10\end{array}$ & $\begin{array}{l}0,73 \\
0,53\end{array}$ & $\begin{array}{l}79 \\
77\end{array}$ & $\begin{array}{l}-4,37 * * \\
-7,70 * *\end{array}$ & $\begin{array}{l}1,33 \\
4,16^{* *}\end{array}$ \\
\hline $\begin{array}{l}\text { sop15/sop69-Unterbrechungen im Gespräch } \\
\text { kann ich unterbinden }\end{array}$ & $\begin{array}{l}\mathbf{0 , 7 4} \\
\mathbf{0 , 3 3}\end{array}$ & $\begin{array}{l}0,32 \\
0,53\end{array}$ & 0,18 & $\begin{array}{l}0,15 \\
0,14\end{array}$ & - & 0,20 & $\begin{array}{l}68,07 \\
66,48\end{array}$ & $\begin{array}{l}19,15 \\
19,15\end{array}$ & $\begin{array}{l}0,69 \\
0,50\end{array}$ & $\begin{array}{l}69 \\
66\end{array}$ & $\begin{array}{l}-4,32 * * \\
-4,24 * *\end{array}$ & $\begin{array}{l}0,59 \\
0,40\end{array}$ \\
\hline $\begin{array}{l}\text { sop10/sop35-Es fällt mir leicht, kritische } \\
\text { Einschätzungen gegenüber Kollegen, Mitar- } \\
\text { beitern etc. nachvollziehbar zu begründen }\end{array}$ & $\begin{array}{l}\mathbf{0 , 7 1} \\
\mathbf{0 , 7 3}\end{array}$ & $\begin{array}{l}0,31 \\
0,33\end{array}$ & $\begin{array}{l}0,28 \\
0,31\end{array}$ & 0,17 & 0,12 & 0,10 & $\begin{array}{l}69,00 \\
65,84\end{array}$ & $\begin{array}{l}18,94 \\
16,59\end{array}$ & $\begin{array}{l}0,74 \\
0,68\end{array}$ & $\begin{array}{l}69 \\
66\end{array}$ & $\begin{array}{l}-3,29 * * \\
-4,83 * *\end{array}$ & $\begin{array}{l}-0,01 \\
1,77\end{array}$ \\
\hline \multicolumn{13}{|l|}{ Motivation von Anderen (5) 14,27\% bzw. $17,81 \%$} \\
\hline $\begin{array}{l}\text { sop05/sop16-Andere (Kollegen, Mitarbeiter, } \\
\text { Kunden, Geschäfts-partner etc.) zu heraus- } \\
\text { ragenden Leistungen anzuspornen ist eine } \\
\text { meiner Stärken }\end{array}$ & $\begin{array}{l}0,33 \\
0,13\end{array}$ & $\begin{array}{l}\mathbf{0 , 8 1} \\
\mathbf{0 , 8 3}\end{array}$ & $\begin{array}{l}0,20 \\
0,14\end{array}$ & $\begin{array}{l}0,23 \\
0,30\end{array}$ & - & - & $\begin{array}{l}61,74 \\
64,43\end{array}$ & $\begin{array}{l}22,07 \\
18,99\end{array}$ & $\begin{array}{l}0,87 \\
0,85\end{array}$ & $\begin{array}{l}62 \\
64\end{array}$ & $\begin{array}{l}-4,35 * * \\
-3,70 * *\end{array}$ & $\begin{array}{l}1,28 \\
-0,26\end{array}$ \\
\hline $\begin{array}{l}\text { sop07/sop21-Es fällt mir leicht, andere (Kol- } \\
\text { legen, Mitarbeiter etc.) entsprechend ihren } \\
\text { Fähigkeiten zu fördern bzw. zu unterstützen }\end{array}$ & 0,23 & $\begin{array}{l}\mathbf{0 , 7 4} \\
\mathbf{0 , 7 6}\end{array}$ & $\begin{array}{l}0,29 \\
0,28\end{array}$ & $\begin{array}{l}0,23 \\
0,24\end{array}$ & - & - & $\begin{array}{l}66,36 \\
69,94\end{array}$ & $\begin{array}{l}18,11 \\
17,47\end{array}$ & $\begin{array}{l}0,77 \\
0,76\end{array}$ & $\begin{array}{l}66 \\
70\end{array}$ & ** & $\begin{array}{l}3,47 * * \\
1,31\end{array}$ \\
\hline $\begin{array}{l}\text { sop14/sop52-Eine Arbeitsatmosphäre zu } \\
\text { schaffen, in der zielge-richtete Aktivität } \\
\text { geschätzt und belohnt wird, ist eine meiner } \\
\text { Stärken }\end{array}$ & $\begin{array}{l}0,22 \\
0,22\end{array}$ & $\begin{array}{l}0,73 \\
0,65\end{array}$ & $\begin{array}{l}0,27 \\
0,35\end{array}$ & $\begin{array}{l}0,15 \\
0,11\end{array}$ & - & 0,15 & $\begin{array}{l}67,87 \\
70,38\end{array}$ & & $\begin{array}{l}0,77 \\
0,68\end{array}$ & $\begin{array}{l}68 \\
70\end{array}$ & & \\
\hline $\begin{array}{l}\text { sop06/sop20-Andere (Kollegen, Mitarbeiter, } \\
\text { Kunden, Geschäftspartner etc.) für eine Idee } \\
\text { etc. zu begeistern fällt mir leicht }\end{array}$ & $\begin{array}{l}0,35 \\
0,21\end{array}$ & $\begin{array}{l}\mathbf{0 , 7 3} \\
\mathbf{0 , 7 7}\end{array}$ & 0,19 & $\begin{array}{l}0,36 \\
0,29\end{array}$ & - & 0,18 & $\begin{array}{l}67,21 \\
67,24\end{array}$ & $\begin{array}{l}17,43 \\
18,14\end{array}$ & $\begin{array}{l}0,81 \\
0,80\end{array}$ & $\begin{array}{l}67 \\
67\end{array}$ & & $\begin{array}{l}1,54 \\
0,04\end{array}$ \\
\hline $\begin{array}{l}\text { sop16/sop55-Andere (Kollegen etc.) dazu } \\
\text { zu befähigen, hervorragende Leistungen zu } \\
\text { erbringen, ist eine meiner Stärken }\end{array}$ & $\begin{array}{l}0,33 \\
0,16\end{array}$ & $\begin{array}{l}\mathbf{0 , 7 2} \\
\mathbf{0 , 8 6}\end{array}$ & $\begin{array}{l}0,21 \\
0,14\end{array}$ & $\begin{array}{l}0,19 \\
0,19\end{array}$ & $-0,19$ & - & $\begin{array}{l}60,53 \\
65,03\end{array}$ & $\begin{array}{l}22,31 \\
18,49\end{array}$ & $\begin{array}{l}0,85 \\
0,82\end{array}$ & $\begin{array}{l}61 \\
65\end{array}$ & & $\begin{array}{l}0,87 \\
0,25\end{array}$ \\
\hline \multicolumn{13}{|c|}{ Akzeptanz und Anerkennung (5) 12,15\% bzw. 13,80\% } \\
\hline $\begin{array}{l}\text { sop12/sop46-Andere Meinungen (von Kol- } \\
\text { legen, Mitarbeitern, Geschäftspartnern etc.) } \\
\text { zu berücksichtigen fällt mir leicht }\end{array}$ & $\begin{array}{l}0,23 \\
0,21\end{array}$ & $\begin{array}{l}0,19 \\
0,18\end{array}$ & $\begin{array}{l}\mathbf{0 , 7 5} \\
\mathbf{0 , 8 0}\end{array}$ & 0,22 & $\begin{array}{l}-0,17 \\
-0,16\end{array}$ & 0,18 & $\begin{array}{l}72,64 \\
73,43\end{array}$ & $\begin{array}{l}15,20 \\
15,51\end{array}$ & $\begin{array}{l}0,81 \\
0,76\end{array}$ & $\begin{array}{l}73 \\
73\end{array}$ & $\begin{array}{l}-2,69 * * \\
-4,58 * *\end{array}$ & $\begin{array}{l}1,26 \\
0,18\end{array}$ \\
\hline $\begin{array}{l}\text { sop13/sop48-Es fällt mir leicht, andere (Kol- } \\
\text { legen, Mitarbeiter, Vorgesetzte, Geschäfts- } \\
\text { partner etc.) so zu akzeptieren, wie sie sind }\end{array}$ & - & - & $\begin{array}{l}\mathbf{0 , 7 2} \\
\mathbf{0 , 7 7}\end{array}$ & - & $\begin{array}{l}-0,21 \\
-0,10\end{array}$ & - & $\begin{array}{l}71,00 \\
71,87\end{array}$ & $\begin{array}{l}18,20 \\
19,04\end{array}$ & $\begin{array}{l}0,59 \\
0,58\end{array}$ & $\begin{array}{l}72 \\
72\end{array}$ & $\begin{array}{l}-3,86 * * \\
-5,06 * *\end{array}$ & $\begin{array}{l}0,43 \\
0,21\end{array}$ \\
\hline $\begin{array}{l}\text { sop08/sop26-Auf Vorschläge anderer (Kol- } \\
\text { legen, Mitarbeiter, Kunden, Geschäftspart- } \\
\text { ner etc.) einzugehen fällt mir leicht }\end{array}$ & $\begin{array}{l}0,21 \\
0,10\end{array}$ & $\begin{array}{l}0,26 \\
0,25\end{array}$ & $\begin{array}{l}0,69 \\
0,72\end{array}$ & $\begin{array}{l}0,16 \\
0,14\end{array}$ & $-0,13$ & 0,14 & $\begin{array}{l}76,36 \\
73,58\end{array}$ & $\begin{array}{l}14,80 \\
15,53\end{array}$ & $\begin{array}{l}0,68 \\
0,65\end{array}$ & $\begin{array}{l}76 \\
74\end{array}$ & $\begin{array}{l}-2,81 * * \\
-4,71 * *\end{array}$ & $\begin{array}{l}-0,31 \\
0,11\end{array}$ \\
\hline $\begin{array}{l}\text { sop11/sop44-Auf die Bedürfnisse anderer } \\
\text { (Kollegen, Mitarbeiter, Geschäftspartner } \\
\text { etc.) einzugehen ist eine meiner Stärken }\end{array}$ & $\begin{array}{l}0,13 \\
0,16\end{array}$ & $\begin{array}{l}0,40 \\
0,33\end{array}$ & $\begin{array}{l}0,68 \\
0,66\end{array}$ & $\begin{array}{l}0,29 \\
0,25\end{array}$ & $\begin{array}{l}-0,11 \\
-0,11\end{array}$ & - & $\begin{array}{l}68,50 \\
73,76\end{array}$ & $\begin{array}{l}19,34 \\
16,05\end{array}$ & $\begin{array}{l}0,76 \\
0,68\end{array}$ & $\begin{array}{l}69 \\
74\end{array}$ & $\begin{array}{l}-3,49 * * \\
-4,85 * *\end{array}$ & $\begin{array}{l}0,13 \\
0,25\end{array}$ \\
\hline $\begin{array}{l}\text { sop17/sop57-Ich kann mich gut auf andere } \\
\text { einstellen }\end{array}$ & 0,10 & $\begin{array}{l}0,30 \\
0,28\end{array}$ & $\begin{array}{l}\mathbf{0 , 6 0} \\
\mathbf{0 , 6 8}\end{array}$ & $\begin{array}{l}0,34 \\
0,31\end{array}$ & $\begin{array}{l}-0,26 \\
-0,12\end{array}$ & 0,11 & $\begin{array}{l}71,74 \\
77,67\end{array}$ & $\begin{array}{l}18,35 \\
15,24\end{array}$ & $\begin{array}{l}0,71 \\
0,67\end{array}$ & $\begin{array}{l}77 \\
78\end{array}$ & $\begin{array}{l}-4,61 * * \\
-4,60 * *\end{array}$ & $\begin{array}{l}3,99 * * \\
-0,45\end{array}$ \\
\hline
\end{tabular}

Itemnummer im KRI/Itemnummer in der Vorversion des KRI. (i) steht für negativ gepoltes Item. Der obere/erste Wert stellt die Ergebnisse in der ersten Stichprobe $(N=140)$, der untere/zweite Wert die Ergebnisse in der zweiten Stichprobe $(N=343)$ dar. Angegeben werden die Varimaxrotierten Faktorladungen der Items und ihre Zuordnung zu den Faktoren. Fett gedruckt sind die Ladungen, die für die Faktorinterpretation herangezogen wurden. Ladungen von $a<0,10$ sind nicht ausgewiesen. In Klammern hinter den Skalenüberschriften steht die Anzahl der Items, dahinter wird die erklärte Varianz nach Rotation für den Faktor angegeben. Die Rotation ist in beiden Stichproben in 6 Iterationen konvergiert 
Tab. 6 (Fortsetzung): EFA zur Faktorenstruktur der Sozialkompetenz

\begin{tabular}{|c|c|c|c|c|c|c|c|c|c|c|c|c|}
\hline \multicolumn{13}{|c|}{ Kontaktaufbau und -pflege (4) 10,84\% bzw. 13,09\% } \\
\hline $\begin{array}{l}\text { sop01/sop01-Es fällt mir leicht, auf andere } \\
\text { (Kollegen, Mitarbeiter, Kunden oder Ge- } \\
\text { schäftsarbeiter) zuzugehen }\end{array}$ & $\begin{array}{l}0,14 \\
0,11\end{array}$ & $\begin{array}{l}0,23 \\
0,16\end{array}$ & $\begin{array}{l}0,25 \\
0,12\end{array}$ & $\begin{array}{l}\mathbf{0 , 8 0} \\
\mathbf{0 , 7 9}\end{array}$ & - & - & $\begin{array}{l}79,64 \\
76,10\end{array}$ & $\begin{array}{l}18,44 \\
20,63\end{array}$ & $\begin{array}{l}0,82 \\
0,64\end{array}$ & $\begin{array}{l}80 \\
76\end{array}$ & $\begin{array}{l}-6,01 * * \\
-9,06 * *\end{array}$ & $\begin{array}{l}3,60 * * \\
4,46 * *\end{array}$ \\
\hline $\begin{array}{l}\text { sop03/sop02-Neue Arbeitskontakte zu knüp- } \\
\text { fen gehört zu meinen Stärken }\end{array}$ & 0,30 & $\begin{array}{l}0,19 \\
0,36\end{array}$ & 0,28 & $\begin{array}{l}\mathbf{0 , 7 3} \\
\mathbf{0 , 8 0}\end{array}$ & $-0,15$ & - & $\begin{array}{l}73,36 \\
69,53\end{array}$ & $\begin{array}{l}19,62 \\
21,39\end{array}$ & $\begin{array}{l}0,79 \\
0,78\end{array}$ & $\begin{array}{l}73 \\
70\end{array}$ & $\begin{array}{l}-3,43 * * \\
-5,10 * *\end{array}$ & $\begin{array}{l}1,11 \\
-0,69\end{array}$ \\
\hline $\begin{array}{l}\text { sop18/sop74-Es fällt mir leicht Gespräche } \\
\text { zu beginnen }\end{array}$ & $\begin{array}{l}0,41 \\
0,16\end{array}$ & $\begin{array}{l}0,24 \\
0,38\end{array}$ & 0,27 & $\begin{array}{l}\mathbf{0 , 7 2} \\
\mathbf{0 , 6 0}\end{array}$ & $-0,19$ & - & $\begin{array}{l}75,29 \\
72,80\end{array}$ & $\begin{array}{l}19,02 \\
21,67\end{array}$ & $\begin{array}{l}0,70 \\
0,62\end{array}$ & $\begin{array}{l}75 \\
73\end{array}$ & $\begin{array}{l}-5,56 * * \\
-5,09 * *\end{array}$ & $\begin{array}{l}3,21 * * \\
-1,59\end{array}$ \\
\hline $\begin{array}{l}\text { sop04/sop03-Interne Kontakte in der Orga- } \\
\text { nisation pflegen kann ich gut }\end{array}$ & 0,11 & $\begin{array}{l}0,22 \\
0,18\end{array}$ & $\begin{array}{l}0,41 \\
0,16\end{array}$ & $\begin{array}{l}\mathbf{0 , 6 7} \\
\mathbf{0 , 8 0}\end{array}$ & $-0,16$ & 0,12 & $\begin{array}{l}75,07 \\
72,85\end{array}$ & $\begin{array}{l}18,83 \\
19,78\end{array}$ & $\begin{array}{l}0,71 \\
0,69\end{array}$ & $\begin{array}{l}75 \\
73\end{array}$ & $\begin{array}{l}-4,59 * * \\
-6,25 * *\end{array}$ & $\begin{array}{l}2,25 \\
1,01\end{array}$ \\
\hline \multicolumn{13}{|c|}{ Rücksichtnahme und Besonnenheit (5) 10,06\% bzw. 14,57\% } \\
\hline $\begin{array}{l}\text { son02/son03-Es kann leicht passieren, dass } \\
\text { ich andere (z. B. Kollegen, Mitarbeiter, Kun- } \\
\text { den, Geschäftspartner etc.) von oben herab } \\
\text { behandle. (i) }\end{array}$ & - & - & $\begin{array}{l}-0,21 \\
-0,11\end{array}$ & $-0,11$ & $\begin{array}{l}0,73 \\
0,86\end{array}$ & - & $\begin{array}{l}21,48 \\
28,63\end{array}$ & $\begin{array}{l}21,01 \\
26,98\end{array}$ & $\begin{array}{l}0,60 \\
0,78\end{array}$ & $\begin{array}{l}79 \\
71\end{array}$ & $\begin{array}{l}-6,34 * * \\
-7,88 * *\end{array}$ & $\begin{array}{l}3,23^{* *} \\
0,54\end{array}$ \\
\hline $\begin{array}{l}\text { son05/son } 40 \text {-Es passiert mir leicht, dass ich } \\
\text { andere vor den Kopf stoße. (i) }\end{array}$ & - & - & $\begin{array}{l}-0,29 \\
-0,10\end{array}$ & - & $\begin{array}{l}\mathbf{0 , 7 3} \\
\mathbf{0 , 8 8}\end{array}$ & - & $\begin{array}{l}21,10 \\
30,28\end{array}$ & $\begin{array}{l}20,28 \\
26,38\end{array}$ & $\begin{array}{l}0,60 \\
0,80\end{array}$ & $\begin{array}{l}79 \\
70\end{array}$ & $\begin{array}{l}-6,88 * * \\
-7,32 * *\end{array}$ & $\begin{array}{l}4,38 * * \\
-0,09\end{array}$ \\
\hline $\begin{array}{l}\text { son03/son04-Wenn mich etwas ärgert, kann } \\
\text { ich schnell ausfallend (zu Kollegen, Mit- } \\
\text { arbeitern, Kunden, Geschäftspartnern etc.) } \\
\text { werden. (i) }\end{array}$ & $-0,20$ & $-0,24$ & - & - & $\begin{array}{l}\mathbf{0 , 7 2} \\
\mathbf{0 , 8 2}\end{array}$ & - & $\begin{array}{l}18,90 \\
25,86\end{array}$ & $\begin{array}{l}21,12 \\
26,63\end{array}$ & $\begin{array}{l}0,62 \\
0,70\end{array}$ & $\begin{array}{l}81 \\
74\end{array}$ & $\begin{array}{l}-7,28 * * \\
-9,89 * *\end{array}$ & $\begin{array}{l}4,79 * * \\
3,10 * *\end{array}$ \\
\hline $\begin{array}{l}\text { son01/son01-Kleine Spitzen (gegen Kolle- } \\
\text { gen, Mitarbeitern Kunden, Geschäftspart- } \\
\text { nern etc.) rutschen mir schon mal raus. (i) }\end{array}$ & 0,27 & $-0,14$ & 0,11 & - & $\begin{array}{l}0,68 \\
0,72\end{array}$ & - & $\begin{array}{l}45,35 \\
46,43\end{array}$ & $\begin{array}{l}27,50 \\
28,41\end{array}$ & $\begin{array}{l}0,52 \\
0,60\end{array}$ & $\begin{array}{l}55 \\
54\end{array}$ & $\begin{array}{l}-0,83 \\
-1,18\end{array}$ & $\begin{array}{l}-2,85 * * \\
-4,33 * *\end{array}$ \\
\hline $\begin{array}{l}\text { son04/son27-Ich neige dazu, ständig zu } \\
\text { kritisieren. (i) }\end{array}$ & 0,11 & - & $-0,19$ & - & $\begin{array}{l}\mathbf{0 , 6 7} \\
\mathbf{0 , 8 3}\end{array}$ & - & $\begin{array}{l}19,94 \\
30,08\end{array}$ & $\begin{array}{l}19,01 \\
25,72\end{array}$ & $\begin{array}{l}0,55 \\
0,73\end{array}$ & $\begin{array}{l}80 \\
70\end{array}$ & $\begin{array}{l}-5,70 * * \\
-6,96 * *\end{array}$ & $\begin{array}{l}2,63 * * \\
-0,12\end{array}$ \\
\hline
\end{tabular}

Itemnummer im KRI/Itemnummer in der Vorversion des KRI. (i) steht für negativ gepoltes Item. Der obere/erste Wert stellt die Ergebnisse in der ersten Stichprobe $(N=140)$, der untere/zweite Wert die Ergebnisse in der zweiten Stichprobe $(N=343)$ dar. Angegeben werden die Varimaxrotierten Faktorladungen der Items und ihre Zuordnung zu den Faktoren. Fett gedruckt sind die Ladungen, die für die Faktorinterpretation herangezogen wurden. Ladungen von $a<0,10$ sind nicht ausgewiesen. In Klammern hinter den Skalenüberschriften steht die Anzahl der Items, dahinter wird die erklärte Varianz nach Rotation für den Faktor angegeben. Die Rotation ist in beiden Stichproben in 6 Iterationen konvergiert.

scharf zu bezeichnen (vgl. Tab. 2, 3, 4, 5, 6 und 7). Die meisten Items weisen mittlere Schwierigkeiten auf. In der ersten Stichprobe sind 17, in der zweiten 8 von 80 Items als zu leicht zu bezeichnen (vgl. Tab. 2, 3, 4, 5, 6 und 7). Darüber hinaus zeigen die Tab. 2, 3, 4, 5, 6 und 7, dass die meisten Items eine linksschiefe Verteilung aufweisen.

Reliabilität Die Reliabilitäten erreichen in den beiden Stichproben durchweg ausreichende Werte, die meisten sind als zufriedenstellend zu bezeichnen (vgl. Tab. 8).

Skaleninterkorrelation Die überwiegend - wie angenommen auch facettenübergreifend - positiven Skaleninterkorrelationen variieren in der ersten Stichprobe zwischen $r=-0,04$ (Skala Rücksichtnahme und Besonnenheit und Skala Positionierung des eigenen Standpunktes) und $r=0,67$ (Skala Motivation von Anderen und Skala Mitwirkung) und in der zweiten zwischen $r=0,01$ (Skala Rücksichtnahme und Besonnenheit und Skala Entwicklung von Lösungen) und $r=0,68 ; p<0,01$ (Skala Entwicklung von Lösungen und Skala Konzeptionelles Denken; vgl. Tab. 8).

\section{Facetten des KRI}

Da der Fragebogen die berufliche Handlungskompetenz mit den vier Facetten Fach-, Methoden-, Sozial- und Selbstkompetenz abbilden soll, muss geklärt werden, ob sich die insgesamt 19 Skalen diesen vier Kompetenzfacetten zuordnen lassen. Zur Beantwortung der Fragestellung wird ein strukturprüfendes Verfahren, die konfirmatorische Faktorenanalyse (KFA; Jöreskog 1993), herangezogen. Zur rechnerischen Durchführung wurde das Statistikprogramm AMOS, Version 5.0 (Arbuckle 1995) genutzt. Als beobachtete Variablen gingen die 19 Skalenmittelwerte ein. Für die Analysen wurden die erste und die zweite Stichprobe zu einer Gesamtstichprobe integriert, so dass sich die StichprobengröBe nach listenweisem Fallausschluss auf eine ausreichend große Stichprobe von $N=500$ beläuft (Loehlin 1998; Tabachnick und Fidell 2001).

Überprüft wurden das Vier-Faktoren-Modell der beruflichen Handlungskompetenz mit der Unterscheidung der Fach-, Methoden-, Sozial- und Selbstkompetenz, das DreiFaktoren-Modell mit der Unterscheidung nach FachlichMethodischer-, Sozial- und Selbstkompetenz und das ZweiFaktoren-Modell mit der Unterscheidung nach fachlichen 
Tab. 7 EFA zur Faktorenstruktur der Selbstkompetenz

\begin{tabular}{|c|c|c|c|c|c|c|c|c|c|c|}
\hline & 1 & 2 & 3 & 4 & $\mathrm{M}$ & $\mathrm{SD}$ & $\mathrm{R}_{\mathrm{it}}$ & $P$ & zs & $\mathrm{zK}$ \\
\hline \multicolumn{11}{|l|}{ Mitwirkung (4) 19,87\% bzw. 17,84\% } \\
\hline $\begin{array}{l}\text { sep10/sep22-Gestaltungsmöglichkeiten innerhalb der } \\
\text { Organisation zu nutzen fällt mir leicht }\end{array}$ & $\begin{array}{l}\mathbf{0 , 8 1} \\
\mathbf{0 , 7 0}\end{array}$ & $-0,17$ & $\begin{array}{l}0,21 \\
0,23\end{array}$ & 0,30 & $\begin{array}{l}68,74 \\
70,97\end{array}$ & $\begin{array}{l}20,05 \\
17,06\end{array}$ & $\begin{array}{l}0,75 \\
0,69\end{array}$ & $\begin{array}{l}69 \\
71\end{array}$ & $\begin{array}{l}-4,45^{* *} \\
-4,18^{* *}\end{array}$ & $\begin{array}{l}2,32 \\
-0,30\end{array}$ \\
\hline $\begin{array}{l}\text { sep05/sep19-Es gelingt mir gut, Dinge, die ich mir vor- } \\
\text { nehme, auch innerhalb der Organisation umzusetzen }\end{array}$ & $\begin{array}{l}\mathbf{0 , 7 9} \\
\mathbf{0 , 8 1}\end{array}$ & $\begin{array}{l}-0,13 \\
-0,18\end{array}$ & 0,17 & $\begin{array}{l}0,19 \\
0,18\end{array}$ & $\begin{array}{l}70,15 \\
71,36\end{array}$ & $\begin{array}{l}16,25 \\
15,50\end{array}$ & $\begin{array}{l}0,74 \\
0,76\end{array}$ & $\begin{array}{l}70 \\
71\end{array}$ & $\begin{array}{l}-2,50 \\
-5,58 * *\end{array}$ & $\begin{array}{l}0,68 \\
2,61 * *\end{array}$ \\
\hline $\begin{array}{l}\text { sep07/sep23-Es ist eine meiner Stärken, konkrete Maß- } \\
\text { nahmen zur Umsetzung von Ideen zu planen }\end{array}$ & $\begin{array}{l}0,78 \\
0,67\end{array}$ & $-0,18$ & $\begin{array}{l}0,22 \\
0,25\end{array}$ & 0,11 & $\begin{array}{l}69,33 \\
71,95\end{array}$ & $\begin{array}{l}20,34 \\
17,19\end{array}$ & $\begin{array}{l}0,68 \\
0,61\end{array}$ & $\begin{array}{l}70 \\
72\end{array}$ & $\begin{array}{l}-4,70 * * \\
-6,02 * *\end{array}$ & $\begin{array}{l}1,86 \\
2,68 * *\end{array}$ \\
\hline $\begin{array}{l}\text { sep04/sep14-Es gelingt mir gut, mir günstige Rahmenbe- } \\
\text { dingungen innerhalb der Organisation zu schaffen }\end{array}$ & $\begin{array}{l}\mathbf{0 , 7 7} \\
\mathbf{0 , 8 2}\end{array}$ & $\begin{array}{l}-0,20 \\
-0,17\end{array}$ & - & $\begin{array}{l}0,24 \\
0,10\end{array}$ & $\begin{array}{l}67,48 \\
71,20\end{array}$ & $\begin{array}{l}19,15 \\
17,12\end{array}$ & $\begin{array}{l}0,71 \\
0,68\end{array}$ & $\begin{array}{l}67 \\
71\end{array}$ & $\begin{array}{l}-4,46 * * \\
-5,62 * *\end{array}$ & $\begin{array}{l}1,87 \\
0,95\end{array}$ \\
\hline \multicolumn{11}{|c|}{ Verantwortungsübernahme und Initiative (5) $18,44 \%$ bzw. $17,52 \%$} \\
\hline $\begin{array}{l}\text { sen03/sen17-Ich neige dazu, für Probleme einen Verant- } \\
\text { wortlichen (Schuldigen) zu suchen. (i) }\end{array}$ & - & $\begin{array}{l}0,79 \\
0,79\end{array}$ & - & - & $\begin{array}{l}19,56 \\
32,89\end{array}$ & $\begin{array}{l}21,64 \\
26,13\end{array}$ & $\begin{array}{l}0,67 \\
0,65\end{array}$ & $\begin{array}{l}81 \\
67\end{array}$ & $\begin{array}{l}-7,42 * * \\
-6,52 * *\end{array}$ & $\begin{array}{l}3,39 * * \\
-0,26\end{array}$ \\
\hline $\begin{array}{l}\text { sen01/sen07-Ich neige dazu, mich bei der Arbeit bzw. } \\
\text { im Gespräch mit den Kollegen über die Probleme bei der } \\
\text { Arbeit zu beklagen. (i) }\end{array}$ & $\begin{array}{l}-0,23 \\
-0,14\end{array}$ & $\begin{array}{l}0,77 \\
0,76\end{array}$ & - & - & $\begin{array}{l}26,07 \\
39,72\end{array}$ & $\begin{array}{l}21,58 \\
25,52\end{array}$ & $\begin{array}{l}0,77 \\
0,64\end{array}$ & $\begin{array}{l}75 \\
60\end{array}$ & $\begin{array}{l}-5,99 * * \\
-3,95 * *\end{array}$ & $\begin{array}{l}2,20 \\
-2,45\end{array}$ \\
\hline $\begin{array}{l}\text { sen02/sen08-Ich lasse mich von Fehlschlägen in der Ar- } \\
\text { beit leicht entmutigen. (i) }\end{array}$ & $\begin{array}{l}-0,23 \\
-0,24\end{array}$ & $\begin{array}{l}\mathbf{0 , 7 7} \\
\mathbf{0 , 7 3}\end{array}$ & $-0,11$ & $-0,23$ & $\begin{array}{l}19,41 \\
34,37\end{array}$ & $\begin{array}{l}17,48 \\
26,39\end{array}$ & $\begin{array}{l}0,74 \\
0,64\end{array}$ & $\begin{array}{l}80 \\
66\end{array}$ & $\begin{array}{l}-7,35 * * \\
-5,81 * *\end{array}$ & $\begin{array}{l}5,65 * * \\
-1,41\end{array}$ \\
\hline $\begin{array}{l}\text { sen05/sen38-Ich gerate schnell in eine ablehnende Hal- } \\
\text { tung: „Das bringt doch nichts“. (i) }\end{array}$ & $-0,28$ & $\begin{array}{l}\mathbf{0 , 7 1} \\
\mathbf{0 , 8 0}\end{array}$ & $-0,25$ & - & $\begin{array}{l}17,56 \\
29,35\end{array}$ & $\begin{array}{l}18,38 \\
25,78\end{array}$ & $\begin{array}{l}0,70 \\
0,67\end{array}$ & $\begin{array}{l}83 \\
71\end{array}$ & $\begin{array}{l}-8,46 * * \\
-8,29 * *\end{array}$ & $\begin{array}{l}8,44 * * \\
1,59\end{array}$ \\
\hline $\begin{array}{l}\text { sen04/sen23-Bei Schwierigkeiten kann es mir leicht pas- } \\
\text { sieren, dass ich auf Autoritäten (Vorgesetzte etc.) verwei- } \\
\text { se. (i) }\end{array}$ & $\begin{array}{l}-0,23 \\
-0,15\end{array}$ & $\begin{array}{l}0,66 \\
0,78\end{array}$ & $-0,11$ & - & $\begin{array}{l}21,85 \\
34,19\end{array}$ & $\begin{array}{l}19,78 \\
25,78\end{array}$ & $\begin{array}{l}0,62 \\
0,68\end{array}$ & $\begin{array}{l}79 \\
66\end{array}$ & $\begin{array}{l}-5,82 * * \\
-4,90 * *\end{array}$ & $\begin{array}{l}2,36 \\
-1,55\end{array}$ \\
\hline \multicolumn{11}{|l|}{ Veränderungsinteresse (5) 11,86\% bzw. 18,79\% } \\
\hline $\begin{array}{l}\text { sep08/sep45-Ich habe immer wieder Interesse an neuen } \\
\text { Ideen und Konzepten }\end{array}$ & $\begin{array}{l}0,32 \\
0,18\end{array}$ & $\begin{array}{l}-0,23 \\
-0,13\end{array}$ & $\begin{array}{l}\mathbf{0 , 8 2} \\
\mathbf{0 , 7 8}\end{array}$ & 0,20 & $\begin{array}{l}80,74 \\
82,01\end{array}$ & $\begin{array}{l}14,84 \\
15,90\end{array}$ & $\begin{array}{l}0,86 \\
0,74\end{array}$ & $\begin{array}{l}81 \\
82\end{array}$ & & $\begin{array}{l}0,70 \\
7,06 *\end{array}$ \\
\hline $\begin{array}{l}\text { sep01/sep44-Ich bin immer wieder neugierig auf neue } \\
\text { Arbeitsinhalte }\end{array}$ & $\begin{array}{l}0,27 \\
0,23\end{array}$ & $-0,23$ & $\begin{array}{l}\mathbf{0 , 8 1} \\
\mathbf{0 , 8 0}\end{array}$ & 0,11 & $\begin{array}{l}80,67 \\
80,14\end{array}$ & $\begin{array}{l}15,70 \\
16,32\end{array}$ & $\begin{array}{l}0,78 \\
0,74\end{array}$ & $\begin{array}{l}81 \\
80\end{array}$ & & $\begin{array}{l}0,05 \\
4,27 *\end{array}$ \\
\hline $\begin{array}{l}\text { sep11/sep30-Ich habe immer wieder Lust auf Neues in } \\
\text { der Arbeit }\end{array}$ & $\begin{array}{l}0,16 \\
0,16\end{array}$ & $-0,28$ & $\begin{array}{l}\mathbf{0 , 7 9} \\
\mathbf{0 , 8 0}\end{array}$ & $\begin{array}{l}0,12 \\
0,11\end{array}$ & $\begin{array}{l}81,85 \\
81,47\end{array}$ & $\begin{array}{l}16,85 \\
15,59\end{array}$ & $\begin{array}{l}0,75 \\
0,74\end{array}$ & $\begin{array}{l}82 \\
81\end{array}$ & $\begin{array}{l}4 * * \\
4 * *\end{array}$ & $\begin{array}{l}3,91 * * \\
5,26 * *\end{array}$ \\
\hline $\begin{array}{l}\text { sep02/sep34-Ich begreife erhöhte Anforderung an meinen } \\
\text { Tätigkeitsbereich als Herausforderung }\end{array}$ & $\begin{array}{l}0,27 \\
0,39\end{array}$ & - & $\begin{array}{l}0,67 \\
0,75\end{array}$ & 0,32 & $\begin{array}{l}82,00 \\
80,93\end{array}$ & $\begin{array}{l}13,09 \\
14,75\end{array}$ & $\begin{array}{l}0,67 \\
0,71\end{array}$ & $\begin{array}{l}82 \\
81\end{array}$ & & $\begin{array}{l}1,56 \\
3,72 *\end{array}$ \\
\hline $\begin{array}{l}\text { sep12/sep40-Ich habe immer wieder Interesse an Verän- } \\
\text { derungen innerhalb der Organisation }\end{array}$ & 0,27 & $-0,13$ & $\begin{array}{l}0,67 \\
0,71\end{array}$ & 0,21 & $\begin{array}{l}72,37 \\
71,78\end{array}$ & $\begin{array}{l}21,76 \\
20,13\end{array}$ & $\begin{array}{l}0,55 \\
0,61\end{array}$ & $\begin{array}{l}72 \\
72\end{array}$ & $\begin{array}{l}-6,33 * * \\
-6,06 * *\end{array}$ & $\begin{array}{l}4,47 * * \\
1,16\end{array}$ \\
\hline \multicolumn{11}{|l|}{ Selbstmanagement (4) 9,70\% bzw. 8,84\% } \\
\hline sep06/sep29-Meine eigenen Grenzen kenne ich & $\begin{array}{l}0,18 \\
0,33\end{array}$ & - & - & $\begin{array}{l}0,67 \\
0,78\end{array}$ & $\begin{array}{l}76,81 \\
76,18\end{array}$ & $\begin{array}{l}14,64 \\
18,61\end{array}$ & $\begin{array}{l}0,52 \\
0,47\end{array}$ & $\begin{array}{l}77 \\
76\end{array}$ & $\begin{array}{l}-2,66 * * \\
-7,03 * *\end{array}$ & $\begin{array}{l}-0,92 \\
0,87\end{array}$ \\
\hline $\begin{array}{l}\text { sep03/sep07-Ich habe Vertrauen in meine Person, z. B. } \\
\text { dass ich die Probleme in der Arbeit bewältigen werde }\end{array}$ & $\begin{array}{l}0,31 \\
0,55\end{array}$ & $-0,40$ & 0,42 & 0,64 & $\begin{array}{l}79,26 \\
79,71\end{array}$ & $\begin{array}{l}15,48 \\
15,28\end{array}$ & $\begin{array}{l}0,47 \\
0,38\end{array}$ & $\begin{array}{l}80 \\
80\end{array}$ & $\begin{array}{l}-4,68 * * \\
-8,31 * *\end{array}$ & $\begin{array}{l}2,63 * * \\
8,79 * *\end{array}$ \\
\hline sep09/sep02-Ich kenne meine Schwächen bei der Arbeit & $\begin{array}{l}0,21 \\
0,20\end{array}$ & - & $\begin{array}{l}0,33 \\
0,22\end{array}$ & $\begin{array}{l}\mathbf{0 , 6 4} \\
\mathbf{0 , 8 3}\end{array}$ & $\begin{array}{l}79,56 \\
78,28\end{array}$ & $\begin{array}{l}14,86 \\
16,18\end{array}$ & $\begin{array}{l}0,44 \\
0,49\end{array}$ & $\begin{array}{l}80 \\
78\end{array}$ & $\begin{array}{l}-5,48 * * \\
-7,22 * *\end{array}$ & $\begin{array}{l}5,47 * * \\
3,04 * *\end{array}$ \\
\hline $\begin{array}{l}\text { sep13/sep47-Es gelingt mir gut, mit meinen Kräften so } \\
\text { zu haushalten, dass die Arbeit nicht zu Lasten meiner } \\
\text { psychischen oder physischen Gesundheit geht }\end{array}$ & $\begin{array}{l}0,26 \\
0,37\end{array}$ & $\begin{array}{l}-0,23 \\
-0,17\end{array}$ & $\begin{array}{l}-0,12 \\
0,13\end{array}$ & $\begin{array}{l}\mathbf{0 , 5 3} \\
\mathbf{0 , 2 0}\end{array}$ & $\begin{array}{l}61,48 \\
63,18\end{array}$ & $\begin{array}{l}22,87 \\
23,50\end{array}$ & 0,37 & $\begin{array}{l}61 \\
63\end{array}$ & $\begin{array}{l}-2,72 * * \\
-2,85 * *\end{array}$ & $\begin{array}{l}-1,04 \\
-2,31\end{array}$ \\
\hline
\end{tabular}

Itemnummer im KRI/Itemnummer in der Vorversion des KRI. (i) steht für negativ gepoltes Item. Der obere/erste Wert stellt die Ergebnisse in der ersten Stichprobe $(N=135)$, der untere/zweite Wert die Ergebnisse in der zweiten Stichprobe $(N=339)$ dar. Angegeben werden die Varimaxrotierten Faktorladungen der Items und ihre Zuordnung zu den Faktoren. Fett gedruckt sind die Ladungen, die für die Faktorinterpretation herangezogen wurden. Ladungen von $a<0,10$ sind nicht ausgewiesen. In Klammern hinter den Skalenüberschriften steht die Anzahl der Items, dahinter wird die erklärte Varianz nach Rotation für den Faktor angegeben. Die Rotation ist in 6 bzw. 5 Iterationen konvergiert

(Fach- und Methodenkompetenz) und überfachlichen Kompetenzen (Sozial- und Selbstkompetenz). Zudem werden Modellen mit korrelierten Faktoren Modelle mit orthogonalen Faktoren gegenübergestellt. Zusätzlich wurde getestet, ob die berufliche Handlungskompetenz ein eindimensionales Konstrukt darstellt (Ein-Faktor-Modell). Die $\chi^{2}$ -
Werte fallen für alle getesteten Modelle signifikant aus (vgl. Tab. 9). Da auf diesen Wert die Stichprobengröße und die Modellkomplexität Einfluss nehmen, muss dieses Ergebnismuster nicht zwangsläufig für eine schlechte Modellanpassung sprechen. Wird der $\chi^{2}$-Wert ins Verhältnis zu den Freiheitsgraden gesetzt, resultieren Werte größer 


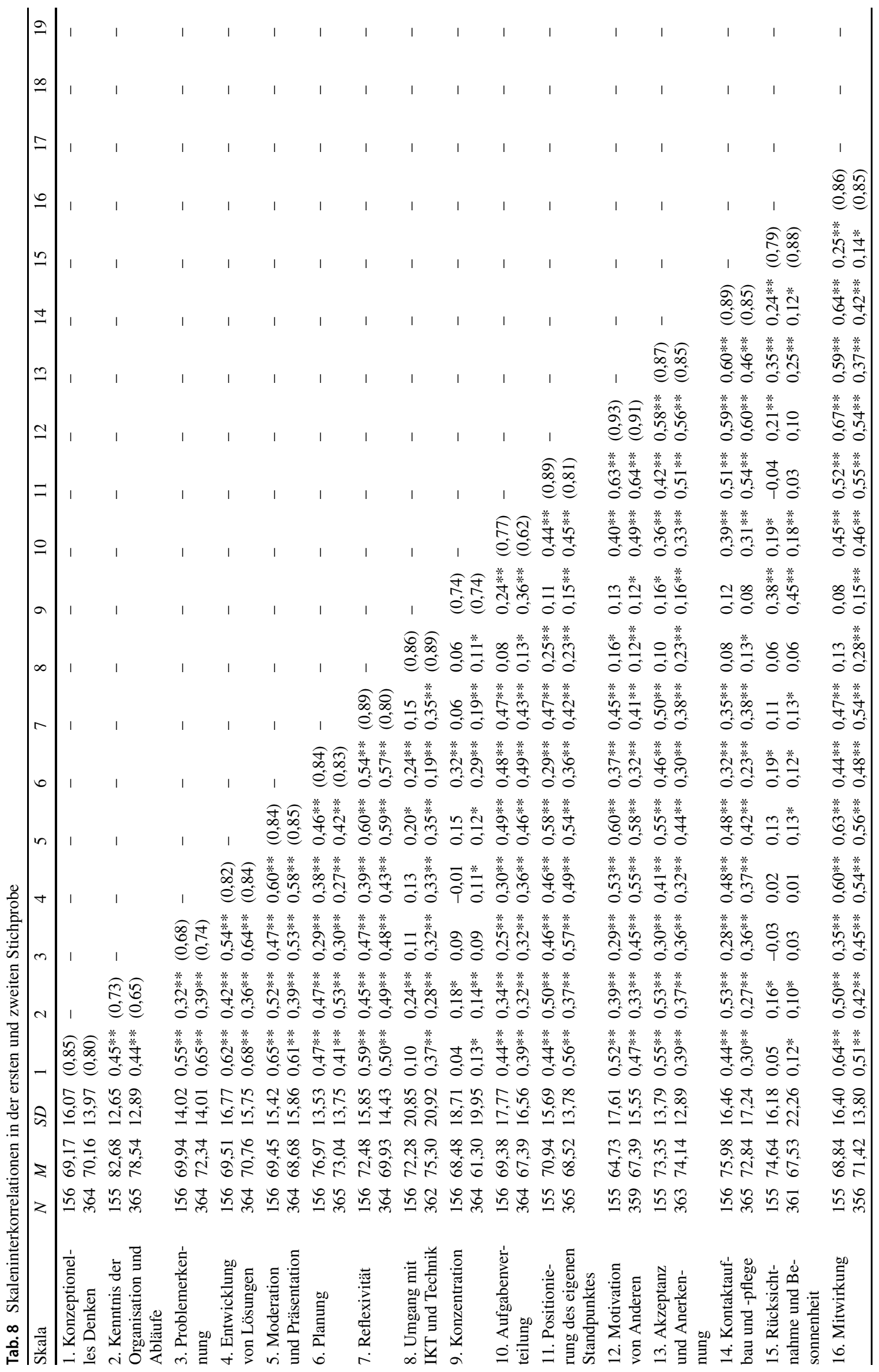




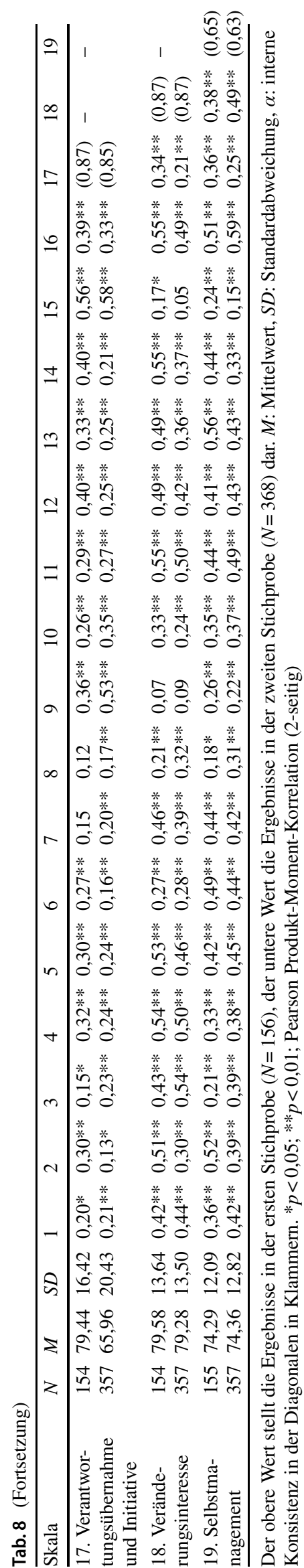

5, wobei das Vier-Faktoren-Modell mit korrelierten Faktoren diesen Wert mit $\chi^{2}=6,90$ am wenigsten übersteigt. Die empfohlenen „FI“-Werte von 0,90 werden von keinem Modell erreicht. Die besten Ergebnisse weisen die korrelierten Zwei-, Drei- und Vier-Faktoren-Modelle auf. Sie nehmen Werte von AGFI $=0,74$ bis GFI $=0,79$ (Zwei-Faktoren-Modell), von AGFI $=0,74$ bis GFI $=0,80$ (Drei-Faktoren-Modell) bzw. AGFI $=0,75$ bis GFI/CFI $=0,81$ (Vier-FaktorenModell) an. Der Grenzwert von RMSEA $=0,10$ wird von den drei Modellen nur knapp überschritten. Wird zwischen den beiden Modellen mit den niedrigsten $\chi^{2}$-Werten (DreiFaktoren-Modell und Vier-Faktoren-Modell) die Verbesserung der Modellanpassung über die $\chi^{2}$-Werte getestet, ergibt sich eine signifikante Modellverbesserung für das Vier-Faktoren-Modell, $\Delta \chi^{2}(3)=69,73 ; p<0,01$. Auf Grund des verhältnismäßig niedrigen $\chi^{2}$-Wertes und Verhältnisses von $\chi^{2}$ Wert zu den Freiheitsgraden, der relativ höheren Fit-Indizes und des Ergebnisses des $\chi^{2}$-Differenz-Tests wird für die berufliche Handlungskompetenz das Vier-Faktoren-Modell, d.h. eine Differenzierung in Fach-, Methoden-, Sozial- und Selbstkompetenz, angenommen.

\subsection{Kriteriumsvalidität}

Die Höhe der Korrelationen zur Validierung des KRI können für beide Stichproben aus Tab. 10 entnommen werden.

Brutto-Jahres-Gehalt Das Brutto-Jahres-Gehalt korreliert wie angenommen auf Facetten- und Skalenebene signifikant positiv mit der Fach- (mit Ausnahme der Skala Problemerkennung), der Methoden- (mit Ausnahme der Skalen Planung, Umgang mit IKT und Technik, Konzentration), der Sozial- und der Selbstkompetenz. Die überwiegend moderaten Korrelationen nehmen Werte zwischen $r=0,40$; $p<0,01$ (Methodenkompetenz, Skala Moderation und Präsentation) und $r=0,19 ; p<0,05$ (Selbstkompetenz, Skala Veränderungsinteresse) an. ${ }^{2}$

Beruflicher Erfolg Die erwarteten Zusammenhänge zum beruflichen Erfolg zeigen sich bei allen Facetten sowie bei den

\footnotetext{
${ }^{2} \mathrm{Da}$ auf das Brutto-Jahres-Einkommen Faktoren wie das Geschlecht oder die Branche Einfluss nehmen (vgl. Statistisches Bundesamt 2021), wurden explorative Analysen zum Unterschied zwischen den Branchen und Geschlechtern hinsichtlich des Brutto-Jahres-Einkommens angeschlossen. Für die Branchenzuordnung ergab eine univariate Varianzanalyse keine signifikanten Unterschiede hinsichtlich des Brutto-Jahres-Einkommens; F=0,94; $p>0,05$. Für das Geschlecht wurde der durchgeführte t-Test signifikant; $t=4,60 ; p<0,01 ; d$. h. Männer verdienen durchschnittlich mehr als Frauen. Auf Grund dieser Ergebnisse wurden für Männer und Frauen getrennte Analysen zum Zusammenhang zwischen dem Brutto-Jahres-Einkommen und den Facetten und Skalen der beruflichen Handlungskompetenz durchgeführt. Die Testung der Differenz zwischen den Korrelationen ergibt, dass sich die Zusammenhänge nicht bedeutsam unterscheiden.
} 
Tab.9 Konfirmatorische Faktorenanalysen zur Faktorenstruktur des KRI

\begin{tabular}{|c|c|c|c|c|c|c|c|c|}
\hline Modell & $\chi^{2}$ & $D f$ & $\chi^{2} / d f$ & GFI & AGFI & NFI & CFI & RMSEA \\
\hline Null-Modell & $4648,53 * *$ & 171 & 27,18 & 0,28 & 0,20 & - & - & 0,23 \\
\hline $\begin{array}{l}\text { Ein-Faktor-Modell der beruflichen Handlungskom- } \\
\text { petenz }\end{array}$ & $1228,20 * *$ & 152 & 8,08 & 0,78 & 0,72 & 0,74 & 0,76 & 0,12 \\
\hline $\begin{array}{l}\text { Zwei-Faktoren-Modell der beruflichen Handlungs- } \\
\text { kompetenz (orthogonale Faktoren) }\end{array}$ & $1599,31 * *$ & 152 & 10,52 & 0,76 & 0,70 & 0,66 & 0,68 & 0,14 \\
\hline $\begin{array}{l}\text { Zwei-Faktoren-Modell der beruflichen Handlungs- } \\
\text { kompetenz (korrelierte Faktoren) }\end{array}$ & $1128,88 * *$ & 151 & 7,48 & 0,79 & 0,74 & 0,76 & 0,78 & 0,11 \\
\hline $\begin{array}{l}\text { Drei-Faktoren-Modell der beruflichen Handlungs- } \\
\text { kompetenz (orthogonale Faktoren) }\end{array}$ & $1899,19 * *$ & 152 & 12,50 & 0,71 & 0,64 & 0,59 & 0,61 & 0,15 \\
\hline $\begin{array}{l}\text { Drei-Faktoren-Modell der beruflichen Handlungs- } \\
\text { kompetenz (korrelierte Faktoren) }\end{array}$ & $1077,54 * *$ & 149 & 7,23 & 0,80 & 0,74 & 0,77 & 0,79 & 0,11 \\
\hline $\begin{array}{l}\text { Vier-Faktoren-Modell der beruflichen Handlungs- } \\
\text { kompetenz (orthogonale Faktoren) }\end{array}$ & $2137,51 * *$ & 152 & 14,06 & 0,67 & 0,59 & 0,54 & 0,56 & 0,16 \\
\hline $\begin{array}{l}\text { Vier-Faktoren-Modell der beruflichen Hand- } \\
\text { lungskompetenz (korrelierte Faktoren) }\end{array}$ & $1007,81 * *$ & 146 & 6,90 & $\mathbf{0 , 8 1}$ & 0,75 & 0,78 & $\mathbf{0 , 8 1}$ & 0,11 \\
\hline
\end{tabular}

$* * p<0,01 . N=500 . d f=$ Freiheitsgrade; GFI = goodness of fit index; $A G F I$ adjusted goodness of fit index; NFI normed fit index; $C F I$ comparative fit index; RMSEA root mean square error of approximation. Fett gedruckt ist das Modell mit dem besten Fit

Tab. 10 Validierungskoeffizienten der Skalen des KRI - Konsequenzen von Kompetenz

\begin{tabular}{|c|c|c|c|c|}
\hline & $\begin{array}{l}\text { Brutto-Jahres- } \\
\text { Gehalt }\end{array}$ & $\begin{array}{l}\text { Beruflicher Er- } \\
\text { folg }\end{array}$ & $\begin{array}{l}\text { Zufriedenheit im } \\
\text { Beruf }\end{array}$ & $\begin{array}{l}\text { Lernrelevante } \\
\text { Merkmale der } \\
\text { Arbeitsaufgabe }\end{array}$ \\
\hline & $N=110$ & $N=154$ & $N=155$ & $N=155$ \\
\hline$\overline{\text { Fachkompetenz }}$ & $0,28 * *$ & $0,51 * *$ & $0,47 * *$ & $0,62 * *$ \\
\hline Konzeptionelles Denken & $0,31 * *$ & $0,41 * *$ & $0,42 * *$ & $0,60 * *$ \\
\hline Kenntnis der Organisation und Abläufe & $0,21 *$ & $0,35^{* *}$ & $0,34 * *$ & $0,40 * *$ \\
\hline Problemerkennung & 0,10 & $0,21 * *$ & $0,28 * *$ & $0,35^{* *}$ \\
\hline Entwicklung von Lösungen & $0,24 *$ & $\begin{array}{l}0,35 * * \\
0,33 * *\end{array}$ & $0,41 * *$ & $0,57 * *$ \\
\hline Methodenkompetenz & $0,32 * *$ & $0,49 * *$ & $0,39 * *$ & $0,43 * *$ \\
\hline Moderation und Präsentation & $0,40 * *$ & $0,39 * *$ & $0,38 * *$ & $0,55^{* *}$ \\
\hline Planung & 0,14 & $0,29 * *$ & $0,33 * *$ & $0,32 * *$ \\
\hline Reflexivität & $0,20 *$ & $0,32 * *$ & $0,30 * *$ & $0,40 * *$ \\
\hline Umgang mit IKT und Technik & 0,12 & 0,11 & 0,07 & 0,05 \\
\hline Konzentration & 0,08 & $0,16^{*}$ & $0,16^{*}$ & 0,01 \\
\hline Aufgabenverteilung & $0,25 * *$ & $0,41 * *$ & $0,30 * *$ & $0,39 * *$ \\
\hline Sozialkompetenz & $0,37 * *$ & $0,53 * *$ & $0,47 * *$ & $0,49 * *$ \\
\hline Positionierung des eigenen Standpunktes & $0,31 * *$ & $0,37 * *$ & $0,27 * *$ & $0,37 * *$ \\
\hline Motivation von anderen & $0,31 * *$ & $0,36 * *$ & $0,33 * *$ & $0,45^{* *}$ \\
\hline Akzeptanz und Anerkennung & $0,32 * *$ & $0,30 * *$ & $0,38 * *$ & $0,37 * *$ \\
\hline Kontaktaufbau und -pflege & $0,22 *$ & $0,41 * *$ & $0,51 * *$ & $0,47 * *$ \\
\hline Rücksichtsnahme und Besonnenheit & $0,22 * *$ & 0,06 & $0,22 * *$ & 0,14 \\
\hline Selbstkompetenz & $0,38 * *$ & $0,61 * *$ & $0,59 * *$ & $0,64 * *$ \\
\hline Mitwirkung & $0,36 * *$ & $0,48 * *$ & $0,54 * *$ & $0,71 * *$ \\
\hline Verantwortungsübernahme und Initiative & $0,28 * *$ & $0,27 * *$ & $0,38 * *$ & $0,35^{* *}$ \\
\hline Veränderungsinteresse & $0,19 *$ & $0,32 * *$ & $0,47 * *$ & $0,48 * *$ \\
\hline Selbstmanagement & $0,32 * *$ & $0,31 * *$ & $0,39 * *$ & $0,36 * *$ \\
\hline
\end{tabular}

$*_{p}<0,05 ; * *<0,01$. Pearson Produkt-Moment-Korrelation (1-seitig) 
Tab. 11 Stationsleitung: Univariate Follow-up Analysen der Skalen der Fachkompetenz

\begin{tabular}{|c|c|c|c|c|}
\hline Skalen der Fachkompetenz & 1. Messzeitpunkt & 2. Messzeitpunkt & $F$ & $\eta^{2}$ \\
\hline Konzeptionelles Denken & $62,15(11,46)$ & $75,00(11,07)$ & $45,16^{* *}$ & 0,54 \\
\hline Kenntnis der Organisation und Abläufe & $79,13(13,46)$ & $87,31(9,94)$ & $22,31 * *$ & 0,36 \\
\hline Problemerkennung & $67,79(11,57)$ & $80,00(8,41)$ & $42,56 * *$ & 0,52 \\
\hline Entwicklung von Lösungen & $64,46(16,88)$ & $78,08(10,91)$ & $29,45^{* *}$ & 0,43 \\
\hline
\end{tabular}

$N=40$. Angeben sind die Mittelwerte und in Klammern die Standardabweichungen, die empirischen F-Werte und die Effektgrößen $\eta^{2}$. ${ }^{*} p<0,05$;

$* * p<0,01, \mathrm{df}=1,39$. Wird eine Korrektur nach Bonferoni durchgeführt, sind die Ergebnisse identisch

Tab. 12 Stationsleitung: Univariate Follow-up Analysen der Methodenkompetenz

\begin{tabular}{|c|c|c|c|c|}
\hline Skalen der Methodenkompetenz & 1. Messzeitpunkt & 2. Messzeitpunkt & $F$ & $\eta^{2}$ \\
\hline Moderation und Präsentation & $62,95(13,89)$ & $77,80(10,75)$ & $64,10 * *$ & 0,62 \\
\hline Planung & $75,63(11,64)$ & $83,56(8,08)$ & $27,89 * *$ & 0,42 \\
\hline Reflexivität & $65,67(14,60)$ & $79,69(9,15)$ & $54,64 * *$ & 0,58 \\
\hline Umgang mit IKT und Technik & $61,83(23,02)$ & $79,19(17,07)$ & $44,15^{* *}$ & 0,53 \\
\hline Konzentration & $66,19(19,17)$ & $79,31(13,23)$ & $13,46 * *$ & 0,26 \\
\hline Aufgabenverteilung & $65,00(17,96)$ & $77,42(13,49)$ & $19,02 * *$ & 0,33 \\
\hline
\end{tabular}

$N=40$. Angeben sind die Mittelwerte und in Klammern die Standardabweichungen, die empirischen F-Werte und die Effektgrößen $\eta^{2}$. ${ }^{*} p<0,05$;

${ }^{* *} p<0,01, \mathrm{df}=1,39$. Wird eine Korrektur nach Bonferoni durchgeführt, sind die Ergebnisse identisch

vier Skalen der Fachkompetenz, bei fünf von sechs Skalen der Methodenkompetenz (Ausnahme: Umgang mit IKT und Technik), bei vier von fünf Skalen der Sozialkompetenz (Ausnahme: Rücksichtnahme und Besonnenheit) und bei den vier Skalen der Selbstkompetenz. Die Korrelationskoeffizienten variieren zwischen $r=0,61 ; p<0,01$ (Selbstkompetenz) und $r=0,16 ; p<0,05$ (Methodenkompetenz, Skala Konzentration).

Zufriedenheit im Beruf Die Zufriedenheit im Beruf weist in der ersten Stichprobe auf Facetten- und Skalenebene bis auf die Skala Umgang mit IKT und Technik (Methodenkompetenz) wie vorhergesagt signifikant positive Zusammenhänge zu den Skalen der beruflichen Handlungskompetenz auf. Die überwiegend moderaten bis mittleren Korrelationen nehmen Werte zwischen $r=0,59 ; p<0,01$ (Selbstkompetenz) und $r=0,16 ; p<0,05$ (Methodenkompetenz, Skala Konzentration) an.

Lernrelevante Merkmale der Arbeitsaufgabe Die lernrelevanten Merkmale der Arbeitsaufgabe stehen mit allen Facetten sowie mit den vier Skalen der Fachkompetenz, vier der sechs Skalen der Methodenkompetenz (Ausnahme: Umgang mit IKT und Technik sowie Konzentration), vier der fünf Skalen der Sozialkompetenz (Ausnahme: Rücksichtnahme und Besonnenheit) und den vier Skalen der Selbstkompetenz bestätigt. Die meist mittleren bis hohen Korrelationen nehmen Werte zwischen $r=0,71 ; p<0,01$ (Selbstkompetenz, Skala Mitwirkung) und $r=0,32 ; p<0,01$ (Methodenkompetenz, Skala Planung) an (vgl. Tab. 10). Besonders starke Zusammenhänge zeigen sich zwischen der Lernförderlichkeit und der Fach- sowie Selbstkompetenz.

\subsubsection{Veränderbarkeit}

Um zu klären, ob mit dem KRI Veränderungen nach Personalentwicklungsmaßnahmen abgebildet werden können, wurden multivariate Varianzanalysen mit univariaten Follow-up Varianzanalysen berechnet.

Studie 1: Stationsleitung In der Teilstichprobe Stationsleitung zeigt sich ein signifikanter Effekt des für den Messwiederholungsfaktor sowohl für die Fach- $(F(4,36)=14,06$, $\left.p<0,01, \eta^{2}=0,61\right)$, die Methoden- $(F(6,34)=11,89, p<0,01$, $\left.\eta^{2}=0,68\right)$, die Sozial- $\left(F(5,34)=5,65, p<0,01, \eta^{2}=0,45\right)$ als auch die Selbstkompetenz $(F(4,35)=7,30, p<0,01$, $\left.\eta^{2}=0,46\right)$. Die univariaten Follow-up Varianzanalysen zeigen eine Verbesserung für alle vier Skalen der Fach-, alle sechs Skalen der Methoden-, vier der fünf Skalen der Sozial- sowie alle vier Skalen der Selbstkompetenz (vgl. Tab. 10, 11, 12 und 13).

Studie 2: Auszubildende Self-Assesment Wie angenommen ergibt sich für die Fachkompetenz in der Studie SelfAssessment kein signifikanter Effekt für den Messwiederholungsfaktor, $F(4,31)=1,87, p>0,05, \eta^{2}=0,19$ und den Interaktionseffekt, $F(4,31)=2,19, p>0,05, \eta^{2}=0,22$. Auch für die Methodenkompetenz ergibt sich kein signifikanter Effekt für den Messwiederholungsfaktor, $F(6$, 29) $=0,92, p>0,05, \eta^{2}=0,16$ und den Interaktionseffekt, $F(6,29)=0,53, p>0,05, \eta^{2}=0,10$. Die Auszubildenden schätzen sich unabhängig von ihrer Gruppenzugehörigkeit hinsichtlich ihrer Fach- und Methodenkompetenz zum ersten Messzeitpunkt genauso ein wie zum zweiten Messzeitpunkt. Entgegen der Annahme ergibt sich bei der 
Tab. 13 Stationsleitung: Univariate Follow-up Analysen der Sozialkompetenz

\begin{tabular}{|c|c|c|c|c|}
\hline Skalen der Sozialkompetenz & 1. Messzeitpunkt & 2. Messzeitpunkt & $F$ & $\eta^{2}$ \\
\hline Positionierung des eigenen Standpunktes & $68,72(14,59)$ & $77,85(12,78)$ & $12,96 * *$ & 0,25 \\
\hline Motivation von anderen & $63,46(13,27)$ & $74,92(13,12)$ & $26,71 * *$ & 0,41 \\
\hline Akzeptanz und Anerkennung & $71,18(12,50)$ & $79,95(13,42)$ & $17,30 * *$ & 0,31 \\
\hline Kontaktaufbau und -pflege & $69,94(15,05)$ & $79,79(18,20)$ & $9,62 * *$ & 0,20 \\
\hline Rücksichtsnahme und Besonnenheit & $68,21(21,14)$ & $72,84(22,40)$ & 1,58 & 0,04 \\
\hline
\end{tabular}

$N=39$. Angeben sind die Mittelwerte und in Klammern die Standardabweichungen, die empirischen F-Werte und die Effektgrößen $\eta^{2}$. $p<0,05$;

$* * p<0,01, \mathrm{df}=1,38$. Wird eine Korrektur nach Bonferoni durchgeführt, sind die Ergebnisse (mit Ausnahme der Skala Kontaktaufbau und

-pflege) identisch. Wird eine Korrektur nach Holm (Holm 1979) durchgeführt, sind die Ergebnisse ohne Ausnahme identisch

Tab. 14 Stationsleitung: Univariate Follow-up Analysen der Selbstkompetenz

\begin{tabular}{|c|c|c|c|c|}
\hline Skalen der Selbstkompetenz & 1. Messzeitpunkt & 2. Messzeitpunkt & $F$ & $\eta^{2}$ \\
\hline Mitwirkung & $66,41(15,24)$ & $78,46(9,96)$ & $29,34 * *$ & 0,44 \\
\hline Verantwortungsübernahme und Initiative & $66,82(16,16)$ & $77,54(18,78)$ & $9,44 * *$ & 0,20 \\
\hline Veränderungsinteresse & $77,22(13,08)$ & $85,95(8,54)$ & $17,88 * *$ & 0,32 \\
\hline Selbstmanagement & $72,93(15,61)$ & $84,81(10,27)$ & $25,78 * *$ & 0,40 \\
\hline
\end{tabular}

$N=39$. Angeben sind die Mittelwerte und in Klammern die Standardabweichungen, die empirischen F-Werte und die Effektgrößen $\eta^{2}$. ${ }^{*} p<0,05$; $* * p<0,01, \mathrm{df}=1,34$. Wird eine Korrektur nach Bonferoni durchgeführt, sind die Ergebnisse (mit Ausnahme der Skala Verantwortungsübernahme und Initiative) identisch. Wird eine Korrektur nach Holm (Holm 1979) durchgeführt, sind die Ergebnisse ohne Ausnahme identisch

Tab. 15 Self-Assessment: Univariate Follow-up Analysen der Sozialkompetenz

\begin{tabular}{|c|c|c|c|c|c|c|}
\hline \multirow[t]{2}{*}{ Skalen der Sozialkompetenz } & \multirow{2}{*}{$\begin{array}{l}\text { Self-Assessment } \\
n=17\end{array}$} & \multirow{2}{*}{$\begin{array}{l}\text { Kein Self-Assessment } \\
n=19\end{array}$} & \multicolumn{2}{|l|}{ Zeit } & \multicolumn{2}{|c|}{ Zeit $\times$ Bedingung } \\
\hline & & & $\bar{F}$ & $\eta^{2}$ & $\bar{F}$ & $\eta^{2}$ \\
\hline \multirow{2}{*}{$\begin{array}{l}\text { Positionierung des eigenen } \\
\text { Standpunktes }\end{array}$} & $60,94(14,85)$ & $63,16(10,74)$ & $9,05 * *$ & 0,21 & 0,03 & 0,00 \\
\hline & $65,29(15,70)$ & $67,05(9,20)$ & & & & \\
\hline \multirow[t]{2}{*}{ Motivation von anderen } & $58,59(14,05)$ & $58,42(15,78)$ & $16,88 * *$ & 0,33 & 1,55 & 0,04 \\
\hline & $62,47(16,10)$ & $65,68(11,99)$ & & & & \\
\hline \multirow[t]{2}{*}{ Akzeptanz und Anerkennung } & $71,41(12,08)$ & $75,26(10,44)$ & 0,91 & 0,03 & 0,84 & 0,02 \\
\hline & $74,12(12,78)$ & $75,32(13,78)$ & & & & \\
\hline \multirow[t]{2}{*}{ Kontaktaufbau und -pflege } & $64,41(16,99)$ & $67,24(15,20)$ & 2,80 & 0,08 & 0,33 & 0,01 \\
\hline & $66,91(17,40)$ & $72,37(13,37)$ & & & & \\
\hline \multirow{2}{*}{$\begin{array}{l}\text { Rücksichtsnahme und } \\
\text { Besonnenheit }\end{array}$} & $67,53(19,53)$ & $78,32(12,49)$ & 0,76 & 0,02 & 0,27 & 0,01 \\
\hline & $71,76(18,08)$ & $79,37(12,77)$ & & & & \\
\hline
\end{tabular}

Der obere Wert stellt die Daten zum ersten Messzeitpunkt, der untere Wert die Daten zum zweiten Messzeitpunkt dar. Angeben sind die Mittelwerte und in Klammern die Standardabweichungen, die empirischen F-Werte und die Effektgrößen $\eta^{2}$. $* p<0,05 ; * * p<0,01, \mathrm{df}=1,34$. Wird eine Korrektur für den Faktor Zeit nach Bonferoni oder Holm (Holm 1979) durchgeführt, sind die Ergebnisse (mit Ausnahme der Skala Positionierung des eigenen Standpunktes) identisch

Sozialkompetenz ein signifikanter Effekt für den Messwiederholungsfaktor, $F(5,30)=3,27, p<0,05, \eta^{2}=0,35$, während der Interaktionseffekt wie angenommen insignifikant bleibt, $F(5,30)=0,72, p>0,05, \eta^{2}=0,11$. In den univariaten Follow-up Varianzanalysen zeigt sich für zwei der fünf Skalen (Positionierung des eigenen Standpunktes und Motivation von anderen) ein signifikanter Effekt für den Messwiederholungsfaktor (vgl. Tab. 15). Da zwischen den Messzeitpunkten nicht nur das Self-Assessment, sondern für beide Gruppen der weitere Fortgang der Ausbildung lag, könnten diese Effekte Ausdruck eines partiellen Ausbildungserfolgs sein. Für die Selbstkompetenz zeigt sich wie angenommen ein signifikanter Effekt für den Messwiederholungsfaktor, $F(4,31)=16,24, p<0,01, \eta^{2}=0,68$ als auch für den Interaktionseffekt, $F(4,31)=24,90, p<0,01$, $\eta^{2}=0,76$. Die univariaten Follow-up Varianzanalysen bestätigen dies für drei von vier Skalen der Selbstkompetenz (vgl. Tab. 16).

\section{Diskussion}

Mit der Entwicklung des KRI wurde explorativ eine Differenzierung innerhalb der Kompetenzfacetten Fach-, Methoden-, Sozial- und Selbstkompetenz aufgezeigt. Die Befunde der explorativen Faktorenanalysen machen deutlich, dass sich eine Differenzierung der Fachkompetenz in die vier Skalen Konzeptionelles Denken, Kenntnis der 
Tab. 16 Self-Assessment: Univariate Follow-up Analysen zur Selbstkompetenz

\begin{tabular}{|c|c|c|c|c|c|c|}
\hline \multirow[t]{2}{*}{ Skalen der Selbstkompetenz } & \multirow{2}{*}{$\begin{array}{l}\text { Self-Assessment } \\
n=17\end{array}$} & \multirow{2}{*}{$\begin{array}{l}\text { Kein Self-Assessment } \\
n=19\end{array}$} & \multicolumn{2}{|l|}{ Zeit } & \multicolumn{2}{|c|}{ Zeit $\times$ Bedingung } \\
\hline & & & $F$ & $\eta^{2}$ & $F$ & $\eta^{2}$ \\
\hline \multirow[t]{2}{*}{ Mitwirkung } & $67,35(13,65)$ & $68,55(12,67)$ & 0,71 & 0,02 & 0,00 & 0,00 \\
\hline & $68,97(13,98)$ & $70,13(11,50)$ & & & & \\
\hline \multirow{2}{*}{$\begin{array}{l}\text { Verantwortungsübernahme und } \\
\text { Initiative }\end{array}$} & $61,65(18,17)$ & $65,26(12,40)$ & $15,35 * *$ & 0,31 & $33,33 * *$ & 0,50 \\
\hline & $82,00(8,51)$ & $61,37(13,97)$ & & & & \\
\hline \multirow[t]{2}{*}{ Veränderungsinteresse } & $68,94(13,23)$ & $66,84(7,87)$ & $67,13 * *$ & 0,66 & $67,13^{* *}$ & 0,66 \\
\hline & $87,06(7,45)$ & $66,84(6,91)$ & & & & \\
\hline \multirow[t]{2}{*}{ Selbstmanagement } & $68,97(13,84)$ & $68,77(10,66)$ & $7,81 * *$ & 0,19 & $48,14 * *$ & 0,59 \\
\hline & $86,18(6,13)$ & $61,45(8,63)$ & & & & \\
\hline
\end{tabular}

Der obere Wert stellt die Daten zum ersten Messzeitpunkt, der untere Wert die Daten zum zweiten Messzeitpunkt dar. Angeben sind die Mittelwerte und in Klammern die Standardabweichungen, die empirischen F-Werte und die Effektgrößen $\eta^{2}$. ${ }^{*} p<0,05 ; * * p<0,01, \mathrm{df}=1,34$. Wird eine Korrektur für den Faktor Zeit nach Bonferoni oder Holm (Holm 1979) durchgeführt, sind die Ergebnisse (mit Ausnahme der Skala Selbstmanagement) identisch. Wird eine Korrektur für den Interaktionseffekt nach Bonferoni durchgeführt, sind die Ergebnisse identisch

Organisation und Abläufe, Problemerkennung, Entwicklung von Lösungen, der Methodenkompetenz in die sechs Skalen Moderation und Präsentation, Planung, Reflexivität, Umgang mit IKT und Technik, Konzentration sowie Aufgabenverteilung, der Sozialkompetenz in die fünf Skalen Positionierung des eigenen Standpunktes, Motivation von anderen, Akzeptanz und Anerkennung, Kontaktaufbau und -pflege, Rücksichtnahme und Besonnenheit und der Selbstkompetenz in die vier Skalen Mitwirkung, Verantwortungsübernahme und Initiative, Veränderungsinteresse, Selbstmanagement vornehmen lässt. Damit lassen sich sowohl die problem-, lösungs- und wissensorientierten Aspekte der Fachkompetenz als auch positive und negative überfachliche Kompetenzaspekte des KKR (Kauffeld 2006a; Kauffeld et al. 2017) wiederfinden. Darüber hinaus weist die Differenzierung innerhalb der Facetten Überschneidungen zu Skalen des smk (Frey und Balzer 2005) wie z.B. Reflexivität, Arbeitstechniken, Zielorientiertes Handeln oder die Verantwortungsbereitschaft auf. Da als Ausgangsbasis der Itemformulierung zudem verschiedene Kompetenzmodelle aus Unternehmen sowie Definitionen der vier Facetten Fach-, Methoden-, Sozial- und Selbstkompetenz genutzt wurden, kann vermutet werden, dass das KRI eine Erfassung wesentlicher Aspekte der Fach-, Methoden-, Sozial- und Selbstkompetenz ermöglicht (vgl. auch Kauffeld und Henschel 2010).

Das KRI greift die in der Literatur verbreitete Differenzierung der beruflichen Handlungskompetenz in vier Kompetenzfacetten auf, die in der KFA tendenziell unterstützt wird. Die Skalen und Facetten des KRI sind nicht unabhängig voneinander, wie die Präferenz eines obliquen Modells sowie die Skaleninterkorrelationen zeigen. Dies entspricht der Annahme, dass die spezifische Ausprägung der beruflichen Handlungskompetenz aus der Verknüpfung der Kompetenzen resultiert (vgl. Kauffeld 2006a; Kauffeld und Paulsen 2018).
Nach der Itemselektion umfasst das KRI 80 Items, die ökonomisch in $20 \mathrm{~min}$ bearbeitet werden können. Die Items des KRI sind weitgehend trennscharf und von mittlerer Schwierigkeit. Durch eine hohe Standardisierung hinsichtlich der Durchführung, Auswertung und Ergebnisinterpretation kann die Objektivität des Instruments als gegeben gelten. Die Skalen des KRI verfügen über sehr gute bis akzeptable interne Konsistenzen (Reliabilität). Mit 80 Items, die in $20 \mathrm{~min}$ beantwortet werden können, und ihrer $\mathrm{Zu}$ sammenfassung in 19 Skalen können Kompetenzen differenziert und ökonomisch zugleich beschrieben werden.

Die ermittelten Zusammenhänge der Skalen des KRI zu verschiedenen subjektiven und objektiven Außenkriterien ergeben erste Hinweise für eine zufriedenstellende kriteriumsbezogenen Validität des Instruments. Die Facetten sowie die meisten der Skalen zur beruflichen Handlungskompetenz weisen moderate bis mittlere Zusammenhänge zur Zufriedenheit im Beruf, dem beruflichen Erfolg sowie dem Brutto-Jahres-Einkommen auf. Lernrelevanten Merkmale der Arbeitsaufgabe stehen in überwiegend mittlerem bis hohem positivem Zusammenhang zu den Kompetenzskalen. Die hohen Korrelationen vor allem zur Fach- und Selbstkompetenz korrespondieren mit den Befunden zu objektiv gemessenen Arbeitsbedingungen und Kompetenzen von Gruppen bei der Bewältigung von Optimierungsaufgaben (Kauffeld 2006b). Lernrelevante Merkmale der Arbeitsaufgabe sind dabei eher Antezedenz als Konsequenz und somit kein Aspekt der Kriteriumvalidität im engeren Sinne. Es sei denn es wird argumentiert, dass hohe Kompetenzen zur lernförderlichen Arbeitsgestaltung im Sinne eines Job-Craftings führen. Dies würde auch die erwarteten hohen Korrelationen der Merkmale lernförderlichen Arbeitsgestaltung zur Selbstkompetenz erklären, dnachgewie im Sinne der diskriminaten Validität interpretiert werden können.

Mit dem KRI lassen sich Veränderungen der beruflichen Handlungskompetenz innerhalb homogener Teilstichpro- 
ben, die durch Personalentwicklungs- und Trainingsmaßnahmen induziert werden, abbilden. Dies zeigt sich zum einen in dem Kompetenzzuwachs von Pflegekräften nach einem Stationsleitungskurs über mehrere Monate. Zum anderen konnte im quasiexperimentellen Design die differentielle Wirksamkeit eines Self-Assessments bei Auszubildenden im Finanzdienstleistungsbereich zur beruflichen Orientierung auf die Selbstkompetenz gezeigt werden. Dies kann als Hinweis für die Veränderungssensitivität und die diskriminante Validität des Instrumentes interpretiert werden.

Damit erfüllt das KRI zentrale Anforderungen, die sich aus dem Kompetenzverständnis, psychometrischen Anforderungen sowie der Praxis ergeben. Für die Forschung liegt damit ein Instrument zur Messung des Selbstkonzepts beruflicher Kompetenz vor, um Forschungsfragestellungen anspruchsvoll zu beantworten.

Für die Praxis steht Organisationen ein Instrument zur Verfügung, mit dem die berufliche Handlungskompetenz der Beschäftigten für Personalentwicklungszwecke ökonomisch erfasst werden kann. Die Kompetenzmessung mit dem KRI stellt einen ersten Schritt zur systematischen Kompetenzentwicklung dar (vgl. Kauffeld 2016; Kauffeld und Paulsen 2018). Durch den Vergleich der Selbsteinschätzungen mit den Einschätzungen zur Bedeutung der Kompetenzaspekte für die Arbeit können sowohl für die Organisation als auch für den Einzelnen Überund Unterforderungen aufgezeigt werden. Kompetenzentwicklungsbedarf wird deutlich und die Ableitung von gezielten Maßnahmen zur Kompetenzentwicklung möglich. Das KRI eignet sich, wie anhand der Veränderungsmessungen gezeigt werden konnte, zur Erfolgsevaluation von Personalentwicklungsmaßnahmen, die von Personalverantwortlichen zunehmend gefordert wird (Kauffeld 2016).

Für den Einzelnen kann das KRI darüber hinaus eine differenzierte Reflexion über die beruflichen Kompetenzen anregen und zu einer realistischeren Einschätzung der eigenen Fähigkeiten und Fertigkeiten beitragen. Damit können angemessene Zielsetzungen generiert werden, deren Verfolgung herausfordernd ist und die damit leistungsförderlich wirken (Zielsetzungstheorie; Locke und Latham 1990). Kompetenzrückmeldungen gilt es mit einer Beratung für die betreffende Person zu verbinden, damit die Rückmeldung nicht unverbindlich im Raum stehen bleibt und in der gegenwärtigen oder künftigen Arbeitstätigkeit handlungsleitend werden kann.

\section{Grenzen}

Die Validität von Selbsteinschätzungen kann durch Attributions- und Beschönigungstendenzen (vgl. Mabe und West 1982) motiviert z. B. durch Bestrebungen nach Selbstwert- schutz oder Selbstwerterhöhung (vgl. Stahlberg et al. 1985) gemindert werden. Daher wurden beim Einsatz des KRI die von Moser (1999) genannten validitätssteigernden Maßnahmen von Selbstbeurteilungen wie identisches Beurteilungsziel für Mitarbeiter und Unternehmen, differenzierte Urteilsdimensionen, verhaltens- statt merkmalsorientierte Beschreibungsebene sowie retrospektive Beurteilung berücksichtigt (vgl. Heidemeier und Moser 2009).

Im Gegensatz zu anderen Selbstbeschreibungsverfahren (z. B. smk, Frey und Balzer 2005), in denen sich die Items oft als zu leicht erweisen, erreichen die KRI-Items mittlere Schwierigkeiten. Die meisten Items sind jedoch, wie bei anderen Kompetenzmessverfahren auch, linksschief. Da die Analysen auf ausreichend großen Stichprobengrößen beruhen ( $n \geq 30$; vgl. Bortz 1999), kann davon ausgegangen werden, dass die Analysen trotz Verletzung der Normalverteilungsannahme zu interpretierbaren Ergebnissen geführt haben.

Die CFA zur Überprüfung der Facetten wurde als ergänzendes Vorgehen eingesetzt. Allerdings sind die Ergebnisse der CFA nur eingeschränkt überzeugend, da zentrale Kennwerte wie der CFI nicht befriedigend sind. Eine mögliche Ursache kann darin liegen, dass verschiedene Kompetenzen innerhalb der Facetten eher kompensatorisch wirken. Zum Beispiel in der Facette die Sozialkompetenz die Positionierung des eigenen Standpunktes ebenso wie Kontaktaufbau und -pflege zur Zielerreichung beitragen.

Die Validierungskriterien basieren - abgesehen vom Brutto-Jahres-Einkommen - auf Selbsteinschätzungen, die ihrerseits der Validierung bedürfen. Die Angaben zur Validität werden als gültig angesehen, weil die Items in Anlehnung an erprobte Instrumente formuliert wurden und akzeptable psychometrische Gütekriterien aufweisen. Darüber hinaus korrespondieren die Ergebnisse mit den Resultaten zum objektiven Kriterium Brutto-Jahres-Einkommen, das als Selbstauskunft erhoben wurde, bei dem die Zusammenhänge eher unter- als überschätzt wurden, da neben der beruflichen Handlungskompetenz der Beschäftigten eine Vielzahl anderer Faktoren (z. B. Bundesland, Branche, Geschlecht) Einfluss auf das durchschnittliche Brutto-JahresEinkommen nimmt. Das Bruttoeinkommen könnte zudem durch spezifischere Unterteilungen von Grundeinkommen, leistungsbezogenen Vergütung und Zusatzvergütungen differenziert werden.

Mit dem KRI wird der linearen Annahme gefolgt, dass ,je mehr Kompetenz ein Stelleninhaber aufweist, desto besser ist es“. In einigen Kompetenzmodellen (vgl. zusammenfassend Kauffeld und Paulsen 2018) wird darauf hingewiesen, dass man von jeder Kompetenz auch zuviel haben kann. Konzeptionelles Denken ist positiv zu bewerten. Die übertriebene Anwendung könnte jedoch in Detailverliebtheit oder Problemorientierung münden. Die Positionierung des eigenen Standpunktes wird von Führungskräften gefor- 
dert, eine zu hohe Orientierung auf Durchsetzung kann zu Akzeptanzverlust bei den Mitarbeitern und Isolation führen etc. Auch wenn sich in der vorliegenden Stichprobe keine Hinweise für kurvilineare Zusammenhänge (Streuungsdiagramme) ergeben haben, sollten sie bei Analysen mit dem KRI berücksichtigt und thematisiert werden.

\section{Ausblick}

Aus Forschungsperspektive stellt das KRI eine Möglichkeit dar, dem Mangel an fundierten Erkenntnissen zum Thema Kompetenz, der vor allem in den unzureichenden Erhebungsmethoden zur Messung von Kompetenz begründet liegt, zu begegnen. $\mathrm{Da}$ in der vorliegenden Arbeit nur eine Auswahl vor allem konstruktnaher Kriterien berücksichtigt werden konnte, sind weitere Untersuchungen zur Validierung des KRI mit objektiven Messverfahren ähnlichen Validitätsanspruches wünschenswert. Für den Praxiseinsatz bieten die Skalen des KRI Ansatzpunkte für die Ableitung von Kompetenzentwicklungsmaßnahmen. Das KRI ist als digitale Variante in das Kompetenz-Navi integrierbar, um es einem größeren Anwenderkreis zugänglich zu machen (Kortsch et al. 2018).

Funding Open Access funding enabled and organized by Projekt DEAL.

Open Access Dieser Artikel wird unter der Creative Commons Namensnennung 4.0 International Lizenz veröffentlicht, welche die Nutzung, Vervielfältigung, Bearbeitung, Verbreitung und Wiedergabe in jeglichem Medium und Format erlaubt, sofern Sie den/die ursprünglichen Autor(en) und die Quelle ordnungsgemäß nennen, einen Link zur Creative Commons Lizenz beifügen und angeben, ob Änderungen vorgenommen wurden.

Die in diesem Artikel enthaltenen Bilder und sonstiges Drittmaterial unterliegen ebenfalls der genannten Creative Commons Lizenz, sofern sich aus der Abbildungslegende nichts anderes ergibt. Sofern das betreffende Material nicht unter der genannten Creative Commons Lizenz steht und die betreffende Handlung nicht nach gesetzlichen Vorschriften erlaubt ist, ist für die oben aufgeführten Weiterverwendungen des Materials die Einwilligung des jeweiligen Rechteinhabers einzuholen.

Weitere Details zur Lizenz entnehmen Sie bitte der Lizenzinformation auf http://creativecommons.org/licenses/by/4.0/deed.de.

\section{Literatur}

Arbuckle, J.L. (1995). Amos users' guide. Chicago: SmallWaters.

Arthur, W. J., Bennett, W. J., Edens, P. S., \& Bell, S. T. (2003). Effectiveness of training in organizations: A meta-analysis of design and evaluation features. Journal of Applied Psychology, 88, 234-245.

Backhaus, K., Erichson, B., Plinke, W., \& Weiber, R. (2000). Multivariate Analysemethoden. Eine anwendungsorientierte Einführung (9. Aufl.). Berlin: Springer.

Bartram, D. (2005). The great eight competencies: a criterion-centric approach to validation. Journal of Applied Psychology, 90(6), 1185-1203. https://doi.org/10.1037/0021-9010.90.6.1185.
Bergmann, B. (2000). Arbeitsimmanente Kompetenzentwicklung. In Arbeitsgemeinschaft Qualifikations-Entwicklungs-Management (Hrsg.), Kompetenzentwicklung und Berufsarbeit (S. 11-40). Münster: Waxmann.

Bortz, J. (1999). Statistik für Sozialwissenschaftler. Bd. 5. Berlin, Heidelberg: Springer.

Decius, J., Schaper, N., \& Seifert, A. (2019). Informal workplace learning: development and validation of a measure. Human Resource Development Quarterly, 30(4), 495-535.

Erpenbeck, J., \& Heyse, V. (1999). Die Kompetenzbiographie. Strategien der Kompetenzentwicklung durch selbst organisiertes Lernen und multimediale Kommunikation. Edition QUEM, Bd. 10. Münster: Waxmann.

Erpenbeck, J., \& v. Rosenstiel, L. (2003). Handbuch Kompetenzmessung. Stuttgart: Schäffer-Poeschel.

Erpenbeck, J., Rosenstiel, L. L., Grote, S., \& Sauter, W. (2017). Handbuch Kompetenzmessung. Erkennen, verstehen und bewerten von Kompetenzen in der betrieblichen, pädagogischen und psychologischen Praxis (3. Aufl.). Stuttgart: Schäffer-Poeschel-Verlag.

Frey, A., \& Balzer, L. (2005). Der Beurteilungsbogen smk: Ein Messverfahren für die Diagnose von sozialen und methodischen Fähigkeitskonzepten. In A. Frey, R. S. Jäger \& U. Renold (Hrsg.), Kompetenzdiagnostik - Theorien und Methoden zur Erfassung und Bewertung von beruflichen Kompetenzen (S. 31-56). Landau: Verlag Empirische Pädagogik.

Frieling, E., Grote, S., \& Kauffeld, S. (2003). Teiltätigkeitslisten als Methode der Kompetenzeinschätzung. In J. Erpenbeck \& L. v. Rosenstiel (Hrsg.), Handbuch Kompetenzmessung (S. 169-184). Stuttgart: Schäffer-Poeschel.

Gerken, M., Beausaert, S., \& Segers, M. (2016). Working on professional development of faculty staff in higher education: Investigating the relationship between social informal learning activities and employability. Human Resource Development International, 19(2), 135-151.

Grohmann, A., Schulte, E. M., \& Kauffeld, S. (2021). Entwicklung und Erprobung eines Kurzfragebogens zur Beurteilung von Trainer/ innenkompetenzen in beruflichen Weiterbildungsmaßnahmen. Gruppe. Interaktion. Organisation. Zeitschrift für Angewandte Organisationspsychologie (GIO), 52(1), 79-90. https://doi.org/ 10.1007/s11612-021-00562-0. Download frei verfügbar.

Grote, S., Kauffeld, S., \& Frieling, E. (Hrsg.). (2012). Kompetenzmanagement: Grundlagen und Praxisbeispiele (2. Aufl.). Stuttgart: Schäffer-Poeschel.

Hacker, W. (1998). Allgemeine Arbeitspsychologie. Psychische Regulation von Arbeitstätigkeiten. Bern: Huber.

HayGroup (2004). Schließen der „Pay-for-performance“-Lücke. Arbeitspapier. http://www.haygroup.de/Bibliothek/ArbeitspapierSchließen_der_Pay-for-performance_Lucke.pdf. Zugegriffen: 10. Juli 2005.

Heidemeier, H., \& Moser, K. (2009). Self-other agreement in job performance ratings: a meta-analytic test of a process model. Journal of Applied Psychology, 94(2), 353.

Helmrich, R., Tiemann, M., Troltsch, K., Lukowski, F., Neuber-Pohl, C., \& Lewalder, A.C. (2016). Digitalisierung der Arbeitslandschaften Keine Polarisierung der Arbeitswelt, aber beschleunigter Strukturwandel und Arbeitsplatzwechsel. Wissenschaftliche Diskussionspapiere. (S. 80). : Bundesinstitut für Berufsbildung.

Herold, G. (2003). Wege aus der Unselbstständigkeit - Selbstevaluation als Instrument, die erlernte Unselbstständigkeit zu verlernen. In F. Elster, Z. Dippl \& G. Zimmer (Hrsg.), Wer bestimmt den Lernerfolg? (S. 95-112). Bielefeld: Bertelsmann.

Holm, S. (1979). A simple sequentially rejective multiple test procedure. Scandinavian Journal of Statistics, 6, 65-70.

Jöreskog, K. B. (1993). Testing structural equation models. In K. A. Bollen \& J.S. Long (Hrsg.), Testing structural equation models (S. 294-316). Newbury Park: SAGE.

Judge, T. A., \& Bono, J.E. (2001). Relationship of core self-evaluation traits-self-esteem, generalized self-efficacy, locus of con- 
trol, and emotional stability - with job satisfaction and job performance: A meta-analysis. Journal of Applied Psychology, 86, $80-92$.

Judge, T. A., Cable, D. M., Boudreau, J. W., \& Bretz Jr., R. D. (1995). An empirical investigation of the predictors of executive career success. Personnel Psychology, 48, 485-519.

Kaiser, H.F. (1958). The varimax criterion for analytic rotation in factor analysis. Psychometrika, 23, 187-200.

Kanning, U.P. (2009). Inventar sozialer Kompetenzen (ISK/ISK-K). Göttingen: Hogrefe.

Kanning, U.P. (2014). Inventar zur Messung sozialer Kompetenzen in Selbst- und Fremdbild (ISK-360 ). Göttingen: Hogrefe.

Kanning, U.P., \& Holling, H. (2002). Handbuch personaldiagnostischer Instrumente. Göttingen: Hogrefe.

Kauffeld, S. (2006a). Kompetenzen messen, bewerten, entwickeln. Ein prozessanalytischer Ansatz für Gruppen. Stuttgart: Schäffer-Poeschel.

Kauffeld, S. (2006b). Self-directed work groups and their impact on team competence. Journal of Occupational and Organizational Psychology, 79, 1-21.

Kauffeld, S. (2016). Nachhaltige Personalentwicklung und Weiterbildung. Betriebliche Seminare und Trainings entwickeln, Erfolge messen, Transfer sichern (2. Aufl.). Berlin: Springer.

Kauffeld, S., \& Albrecht, A. (2021). Kompetenzen und ihre Entwicklung in der Arbeitswelt von Morgen: branchenunabhängig, individualisiert, verbunden, digitalisiert? Gruppe. Interaktion. Organisation. Zeitschrift für Angewandte Organisationspsychologie (GIO), 52(1), 1-6. https://doi.org/10.1007/s11612-021-00564-y. Download frei verfügbar.

Kauffeld, S., \& Henschel, A. (2010). Das Kompetenz-Reflexions-Inven$\operatorname{tar}(K R I)$. In W. Sarges, H. Wottawa \& C. Roos (Hrsg.), Organisationspsychologische Instrumente 2. Aufl. Handbuch wirtschaftspsychologischer Testverfahren, (Bd. II, S. 201-207). Lengerich: Pabst.

Kauffeld, S., \& Paulsen, H. (2018). Kompetenzmanagement in Unternehmen. Kompetenzen beschreiben, messen, entwickeln und nutzen. Stuttgart: Kohlhammer.

Kauffeld, S., Grote, S., \& Frieling, E. (2017). Das Kasseler-Kompetenz-Raster (KKR). In J. Erpenbeck, L. v. Rosenstiel, S. Grote \& J. Sauter (Hrsg.), Handbuch Kompetenzmessung (3. Aufl. S. 326-345). Stuttgart: Schäffer-Poeschel.

Kortsch, T., Paulsen, H., \& Kauffeld, S. (2018). Unterstützungskultur trifft auf digitale Lösungen: Kompetenzentwicklung mit dem KOMPETENZ-NAVI optimieren. In S. Kauffeld \& F. Frerichs (Hrsg.), Kompetenzmanagement in kleinen und mittelständischen Unternehmen: Eine Frage der Betriebskultur? (S. 181-193). Berlin: Springer.

Kortsch, T., Schulte, E. M., \& Kauffeld, S. (2019). Learning @ Work: Informal Learning Strategies of German Craft Workers. European Journal of Training and Development, 43(5/6), 418-434. https:// doi.org/10.1108/EJTD-06-2018-0052.

Kraiger, K., Ford, J., \& Salas, E. (1993). Application of cognitive, skillbased and affective theories of learning outcomes to new methods of training evaluation. Journal of Applied Psychology, 78, 311-328.

Van Laar, E., Van Deursen, A. J., Van Dijk, J. A., \& De Haan, J. (2017). The relation between 21 st-century skills and digital skills: a systematic literature review. Computers in human behavior, 72 , $577-588$.

Locke, E. A., \& Latham, G. P. (1990). A theory of goal setting and task performance. Englewood Cliffs: Prentice Hall.

Loehlin, J.C. (1998). Latent variable models. An introduction to factor, path, and structural analysis (3. Aufl.). Mahwah: Lawrence Erlbaum.

Mabe, P. A., \& West, S. W. (1982). Validity of self-evaluation of ability: review and meta-analysis. Journal of Applied Psychology, 67, 280-296.
Miles, R.E. (1965). Human relations or human resources? Harvard Business Review, 43, 148-163.

Moser, K. (1999). Selbstbeurteilung beruflicher Leistung: Überblick und offene Fragen. Psychologische Rundschau, 50, 14-25.

Reisse, W. (1995). Ein prüfungsspezifischer SchlüsselqualifikationsKatalog. Berufsbildung in Wissenschaft und Praxis, 24(6), 47-50.

Richter, F., \& Wardanjan, B. (2000). Die Lernhaltigkeit der Arbeitsaufgabe - Entwicklung und Erprobung eines Fragebogens zu lernrelevanten Merkmalen der Arbeitsaufgabe (FLMA). Zeitschrift für Arbeitswissenschaft, 54(3-4), 175-183.

Rowold, J., \& Kauffeld, S. (2009). Effects of career-related continuous learning on competencies. Personnel Review, 38(1), 90-101. https://doi.org/10.1108/00483480910920732.

Rudolph, C.W., Katz, I.M., Lavigne, K.N., \& Zacher, H. (2017). Job crafting: a meta-analysis of relationships with individual differences, job characteristics, and work outcomes. Journal of Vocational Behavior, 102, 112-138. https://doi.org/10.1016/j.jvb. 2017.05.008.

Sarges, W., \& Wottawa, H. (2001). Handbuch wirtschaftpsychologischer Testverfahren. Lengerich: Pabst.

Schaper, N. (2000). Gestaltung und Evaluation arbeitsbezogener Lernumgebungen. Heidelberg: Ruprecht-Karls-Universität. Unveröffentlichte Habilitation

Soderquist, E. K., Papalexandris, A., Ioannou, G., \& Prastacos, G. (2010). From task-based to competency-based. Personnel Review, 39(3), 325-346. https://doi.org/10.1108/00483481011030520.

Sonntag, K.H. (2004). Personalentwicklung. In H. Schuler (Hrsg.), Organisationspsychologie - Grundlagen und Personalpsychologie. Enzyklödädie der Psychologie: Themengebiet D Praxisgebiete, Serie III Wirtschafts-, Organisations- und Arbeitspsychologie, (Bd. 3, S. 827-890). Göttingen: Hogrefe.

Sonntag, K.H., \& Schäfer-Rauser, U. (1993). Selbsteinschätzung beruflicher Kompetenzen bei der Evaluation von Bildungsmaßnahmen. Zeitschrift für Arbeits- und Organisationspsychologie, 37, 163-171.

Sonntag, K.H., \& Schaper, N. (1999). Förderung beruflicher Handlungskompetenz. In K.H. Sonntag (Hrsg.), Personalentwicklung in Organisationen (S. 211-244). Göttingen: Hogrefe.

Sonntag, K.H., \& Schmidt-Rathjens, C. (2004). Kompetenzmodelle - Erfolgsfaktoren im HR-Management? Personalführung, 37, $18-26$.

Stahlberg, D., Osnabrügge, G., \& Frey, D. (1985). Die Theorie des Selbstwertschutzes und der Selbstwerterhöhung. In D. Frey \& M. Irle (Hrsg.), Motivations- und Informationsverarbeitungstheorien. Theorien der Sozialpsychologie, (Bd. 3, S. 79-124). Bern: Huber.

Statistisches Bundesamt (2021). Statistisches Jahrbuch 2021. Wiesbaden: DeStatis.

Tabachnick, B. G., \& Fidell, L. S. (2001). Using multivariate statistics (4. Aufl.). Boston: Allyn \& Bacon.

Wardanjan, B. (2000). Berufsbiografie und Kompetenzentwicklung. In B. Bergmann, A. Fritsch, P. Göpfert, F. Richter, B. Wardanjan \& S. Wilczek (Hrsg.), Kompetenzentwicklung und Berufsarbeit (S. 133-179). Münster: Waxmann.

Weinert, F.E. (2001). Concept of competence: a conceptual clarification. In D. Rychen \& L. Salganik (Hrsg.), Defining and selecting key competencies (S. 45-65). Kirkland: Hogrefe \& Huber.

Wieland, R. (2004). Arbeitsgestaltung, Selbstregulationskompetenz und berufliche Kompetenzentwicklung. In B.S. Wiese (Hrsg.), Individuelle Steuerung beruflicher Entwicklung. Kernkompetenzen in der modernen Arbeitswelt (S. 169-196). Frankfurt a. M.: Campus. 


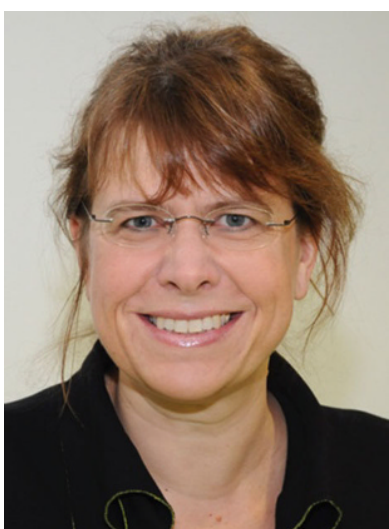

Univ.-Prof. Dr. Simone Kauffeld ist Inhaberin des Lehrstuhls für Arbeit-, Organisations- und Sozialpsychologie der Technischen Universität Braunschweig. In ihrer Forschungstätigkeit setzt sie sich in zahlreichen Projekten mit den Themen Kompetenzentwicklung und -management (Training und Transfer), Team und Führung, Karriere/ Coaching sowie Veränderungen in Organisation und Arbeit auseinander. Das Thema Digitalisierung ist als Querschnittsthema präsent. Als Herausgeberin hat sie die Zeitschriften „PersonalQUARTERLY“ und „Gruppe. Interaktion. Organisation“ neu aufgesetzt und gibt Buchreihen zur Arbeits- und Organisationspsychologie heraus. Um aktiven Wissenstransfer zu leisten, hat sie 2008 die 4A-SIDE GmbH gegründet, die psychologische Expertise mit IT-Kompetenz verbindet. 